\title{
4 \\ Rudolf Carnap: Philosoph der Neuen Sachlichkeit
}

\author{
Hans-Joachim Dahms
}

\subsection{Einleitung}

Der Wiener Kreis war - neben der Frankfurter Schule - diejenige deutschsprachige philosophische Gruppierung in der ersten Hälfte des 20. Jahrhunderts, die im engsten Kontakt zu den wichtigsten zeitgenössischen Kultur- und Kunstströmungen stand. Diese These habe ich, bezogen auf den Wiener Kreis und das Bauhaus in der Zeit des zweiten Bauhausdirektors Hannes Meyer - nicht unter seinem Vorgänger Walter Gropius und erst recht nicht in der Ära seines Nachfolgers Mies van der Rohe -, einmal vertreten (Dahms 2004). ${ }^{1}$ Hier nun möchte ich die Frage an Rudolf Carnap exemplifizieren. Dabei fasse ich das Thema etwas weiter, weil jetzt sein Verhältnis nicht nur zum Bauhaus, sondern zu der gesamten Bewegung der Neuen Sachlichkeit zur Debatte steht. Wenn man seine komplizierten und schwierigen, mit logischen Formeln übersäten Texte liest, wird man nicht leicht auf die Idee kommen, dass dieser „Fugenschreiber der Logik“ (ein Ausdruck von Franz Roh (1925, S. 16), ${ }^{2}$ dem wichtigsten Theoretiker der Neuen Sachlichkeit) großes Interesse an

Für Hinweise, Anregungen und Kritik danke ich Christian Damböck, Christoph Limbeck-Lilienau und Brigitte Parakenings.

Rechtschreibung und Zeichensetzung in Zitaten wurden heutigen Gepflogenheiten angeglichen.

\footnotetext{
${ }^{1}$ Zur Diskussion der These siehe Abschnitt 4.4.

${ }^{2}$ Dort wird der Ausdruck zwar ohne namentliche Nennung eines Trägers verwandt. Aber wenn man an Rohs Freundschaft mit Carnap denkt und auch an den Umstand, dass er sonst keinen namhaften Logiker gekannt hat, dürfte klar sein, wer gemeint war.
}

\section{H.-J. Dahms (殴}

Institute Vienna Circle, University of Vienna, Vienna, Österreich

E-Mail: hans-joachim.dahms@univie.ac.at

C. Damböck, G. Wolters (eds.), Der junge Carnap in historischem Kontext:

1918-1935 / Young Carnap in an Historical Context: 1918-1935,

Veröffentlichungen des Instituts Wiener Kreis 30,

https://doi.org/10.1007/978-3-030-58251-7_4 
zeitgenössischer Kunst und Kultur gehabt haben könnte und im intensiven Austausch mit Kunst- und Architekturtheoretikern und -kritikern stand.

Mein Beitrag hat folgenden Aufbau:

- Im ersten Teil werde ich beschreiben, wann und wo in seiner Vita als Schüler, Student und junger Wissenschaftler schon Beziehungen zu diesen Bereichen zu finden sind;

- im zweiten Teil wende ich mich der Frage zu, welche persönlichen Berührungspunkte er mit der Bewegung der Neuen Sachlichkeit der 1920er-Jahre und ihren Exponenten hatte;

- im dritten Teil schließlich wird besprochen, was seine Philosophie inhaltlich mit der Neuen Sachlichkeit zu tun hat.

\subsection{Carnaps Vita und sein Verhältnis zu Kultur und Kunst}

\subsubsection{Während der Schulzeit}

Carnap wuchs in einer pietistischen Textilfabrikantenfamilie im heutigen Wuppertal auf, die sich in dieser Gegend bis in das Jahr 1550 zurückverfolgen lässt. ${ }^{3}$ Spätestens 1742 entschloss sich die Familie, das Sündenbabel Barmen (aus dem übrigens auch der Textilfabrikantensohn Friedrich Engels stammt) zu verlassen, um sich auf den Höhen der nahe gelegenen Siedlung Ronsdorf sozusagen ein neues Zion zu errichten (Goebel 1970, S. 9 ff.). Dort wurde 1826 Rudolf Carnaps Vater Johann Sebulon geboren. ${ }^{4}$ Er musste wegen der damals noch weit verbreiteten Kinderarbeit die Elementarschule als Zehnjähriger verlassen, nahm aber abends nach der Arbeit Stunden in Schreiben, Geometrie und Zeichnen, und später sogar in Französisch. Er gründete eine Bandfabrik und arbeitete sich im Laufe der Zeit zu erheblichem Reichtum und auch zu großem Ansehen in seiner Heimatgemeinde und deren reformierter Kirche empor, in denen er jeweils jahrelang viele Ehrenämter versah.

Johann Sebulon war dreimal verheiratet und hatte aus diesen Ehen insgesamt 14 Kinder. In dritter Ehe hatte er Carnaps spätere Mutter Anna, geb. Dörpfeld, geheiratet, die nach dem Tod Johann Sebulons ihre beiden Kinder aufzog. Wie in vielen dieser Familien spielte neben einer soliden humanistischen Schulausbildung Hausmusik eine große Rolle; Carnap spielte ausweislich seines Tagebuchs noch in seiner Zeit in Buchenbach in den 1920er-Jahren Cello. ${ }^{5}$ Er wurde auch sonst bei Besuchen

\footnotetext{
${ }^{3}$ Siehe dazu und zur Familiengeschichte Carnaps Goebel (1970, S. 9 ff.).

${ }^{4}$ Carnap hat ihn in Carnap (1963, S. 3) zu ,Johann S.“ abgekürzt. Sebulon war einer der Söhne des biblischen Jakob und der Name eines der Stämme Israels. Die genannten Orte wurden (wie etwa auch Elberfeld, Vohwinkel, Cronenberg und Beyenburg) erst 1929 im Zuge der preußischen Gebietsreform zur Stadt Wuppertal vereinigt.

${ }^{5}$ Siehe dazu seine zahlreichen Tagebucheintragungen zwischen 1920 und 1926. Ich weiß nicht, ob und gegebenenfalls wann er das aufgegeben hat.
} 
im Theater und Konzerthaus mit der Hochkultur vertraut. Sein Gesichtskreis erweiterte sich immer mehr durch eine erstaunliche Reiseaktivität und das Erlernen mehrerer Fremdsprachen.

Anna Carnap war Tochter eines bekannten Schulpädagogen (siehe Beeck 1975) und - das ist hier wichtiger - Schwester von Wilhelm Dörpfeld. Der war von der Ausbildung her eigentlich Architekt. Von dieser Expertise zeugte noch der Umstand, dass er nach dem Tode Johann Sebulons im Jahre 1898 und dem späteren Umzug der Familie nach Jena für seine Schwester dort eine Villa baute. ${ }^{6}$ Berühmt wurde Dörpfeld aber als Archäologe. Als solcher half er Heinrich Schliemann bei der Ausgrabung von Troja, indem er wissenschaftliche und vor allem schonende Ausgrabungstechniken durchsetzte. Danach betätigte er sich bei den Ausgrabungen von Olympia und grub unter eigener Regie Mykene und Tiryns aus. Er wirkte dann viele Jahre als Leiter des Deutschen Archäologischen Instituts in Athen. Auf diese Sachverhalte hat schon André W. Carus (2007, S. 46) hingewiesen. ${ }^{7}$ In seinen späten Jahren widmete sich Dörpfeld zunehmend der Aufgabe, die westgriechische Insel Levkas als das homerische Troja zu erweisen. Voraussetzung für diese These war natürlich, dass er die Dichtungen Homers nicht für bloße Mythen hielt, sondern für dichterisch überhöhte Berichte, die einen historischen Kern hätten. Für weitere Forschungen zu seiner - umstritten gebliebenen - Lieblingsidee legte er auch den Lehrauftrag an der Universität Jena nieder, den er als Honorarprofessor nach dem Ende des Ersten Weltkriegs erhalten hatte. Wegen seiner Verdienste erhielt Dörpfeld ein Dutzend Ehrendoktorhüte im In- und Ausland. Das humanistische Gymnasium in Wuppertal (das ich von 1957 bis 1966 besucht habe) ist wie die Deutsche Schule in Athen nach ihm benannt.

Warum erzähle ich das? Der junge Carnap wuchs in diesem Milieu zwischen Pietismus und Wissenschaft, humanistischer Bildung und aufsehenerregenden archäologischen Ausgrabungsresultaten auf. Schon als Schüler wurde er zweimal von „Onkel Wilhelm“ nach Griechenland eingeladen, nämlich zuerst als 14-Jähriger nach seiner Konfirmation 1905 und dann noch einmal fünf Jahre später nach seinem Abitur. Damals gab es natürlich noch keine Flugzeugverbindungen. Man musste jeweils eine zweitägige Zugreise mit dem Orient-Express machen oder mit dem Zug nach Venedig oder ins italienische Brindisi fahren und von dort mit der Fähre nach Korfu, Igumenitsa, Patras und weiter nach Athen. ${ }^{8}$ Beim ersten Mal scheint Carnap von einer größeren Delegation seiner Familie begleitet worden zu sein (Carus 2007, S. 46), ${ }^{9}$ beim zweiten dann nicht mehr. Carnap hielt sich jeweils nicht nur in Athen auf, sondern besuchte auch die klassischen Stätten. Er stand Dörpfeld, der

\footnotetext{
${ }^{6}$ Sie ist auf der Frontseite des Bandes Carnap Brought Home (Awodey und Klein 2004) im Hintergrund hinter den Mitgliedern der Tagung zu sehen.

${ }^{7}$ Dort wird aber nicht erwähnt, dass es sich um zwei verschiedene Reisen handelte.

${ }^{8}$ Ich gehöre zu den Glücklichen, die als Schüler von der Wilhelm-Dörpfeld-Stiftung eine mehrwöchige Studienreise zu Dörpfelds Grab auf der Insel Levkas und dann weiter zu den klassischen Städten des griechischen Altertums wie Olympia, Sparta, Mykene, Tiryns, Korinth, Delphi, Athen und am Ende nach Kreta spendiert bekamen.

${ }^{9}$ Mit von der Partie war jedenfalls Carnaps ältere Schwester Agnes (siehe Göebel 1970, S. 14).
} 
ihm höchstpersönlich die Höhepunkte Athens gezeigt hatte, sogar bei der Ausgrabung von Tiryns durch Mithilfe bei Vermessungsarbeiten zur Seite. ${ }^{10}$ Zeitgenössische Fotos zeigen den jungen Carnap mit einer Meßlatte. ${ }^{11}$

Carnaps Freunde aus Studentenzeiten, Wilhelm Flitner und Franz Roh, schrieben ihm in den 1960er-Jahren eine Ansichtskarte, als sie zum ersten Mal in Athen waren, und erinnerten an Carnaps mehr als 50 Jahre früheren Aufenthalt.

\subsubsection{Studentenjahre}

Flitner und Roh kannten sich schon seit der Schulzeit in Weimar und begannen 1909 gemeinsam ihr Studium in München (Flitner 1986, S. 82, 99). Danach zog Flitner nach Jena und Roh nach Leipzig. Carnap kam 1909 zum Studium der Philosophie, Mathematik und Physik nach Jena. Zusammen wirkte er mit Flitner dann im Jenaer Sera-Kreis, ${ }^{12}$ zu dem Roh häufiger aus Leipzig herüberkam. Der Kreis veranstaltete - oft im großen Haus der Familie Carnap - Lesungen, Diskussionen und Tanzabende. Darüber hinaus betrieb man extramurale Aktivitäten, wie alljährliche Sonnwendfeiern auf den Bergen oberhalb Jenas und Vorführungen von Theaterstücken in den Dörfern der Umgebung. Carnap übernahm bei solchen Gelegenheiten auch Hauptrollen wie etwa den Faust in einer volkstümlichen Fassung des Stoffes. ${ }^{13}$

Eine der prägenden Erfahrungen dürfte das gemeinsam vom Sera-Kreis und vom Deutschen Werkbund veranstaltete „Künstlerfest“ am 7. Juni 1913 gewesen sein, das die Jahrestagung des Werkbunds in Leipzig begleitete (Werner 2003, S. 126-129). Es fand ca. 30 Kilometer nördlich von Jena auf den Wiesen unterhalb der Rudelsburg bei Bad Kösen statt. ${ }^{14}$ Der Anstoß zu dieser gemeinsamen Veranstaltung ging offenbar auf den Jenaer Verleger Eugen Diederichs zurück. Dieser hatte 1907 zu den zwölf Unternehmern gehört, die zusammen mit zwölf Künstlern den Deutschen Werkbund gründeten. Es handelte sich um eine Vereinigung von Designern, Architekten, Industriellen und Verlegern, die sich einer Modernisierung der Architektur, des Designs und der Gebrauchskunst verschrieben hatte und bereits die

\footnotetext{
${ }^{10}$ Da Carnap erst 1908 Tagebücher geschrieben hat, liegt für die erste Reise kein Bericht vor. Siehe für die zweite Reise das Carnap-Tagebuch vom 24. März bis zum 3. Mai 1910; die Vermessungsarbeiten in Tiryns fanden vom 19. bis zum 21. April 1910 statt. Offenbar gibt es von dieser Reise auch einen noch nicht transkribierten ausführlicheren Text Carnaps.

${ }^{11}$ Abgedruckt z. B. in der Wiener-Kreis-Dokumentation von Christoph Limbeck-Lilienau (2015, S. 73).

${ }^{12}$ Siehe zur Heranbildung dieser Kerngruppe des Sera-Kreises Flitner (1986, S. 125 ff.), zu Carnap dort S. $126 \mathrm{f}$.

${ }^{13}$ Siehe dazu die Aufzeichnungen von Martha Hörmann, 15 und 18 in der Sera-Sammlung (ohne Signatur) im Carnap-Nachlass. Sie beziehen sich auf eine Aufführung in der zweiten Juni-Hälfte 1913. Das Tagebuch hat zwischen dem 5. Juni und dem 1. August 1913 eine Lücke.

${ }^{14}$ Siehe zum Ablauf dieser Veranstaltung das Diederichs-Zitat in Werner (2003, S. 127 f.).
} 
„Sachlichkeit“ der Formgebung und einen entsprechenden Funktionalismus propagierte. Diederichs betätigte sich auch als Ideengeber und Sponsor des Sera-Kreises.

Carnaps Tagebuch weist für diese Zeit im Jahre 1913 eine größere Lücke auf, so dass wir nicht über seine Aktionen und Eindrücke bei dem Fest unterrichtet sind. Aber in seinem Nachlass finden sich Aufzeichnungen anderer Teilnehmer, die sowohl einen guten Einblick in den Ablauf der Ereignisse gestatten als auch einen Eindruck von der Begeisterung, mit der die Teilnehmer sie begleiteten. Diesen Augenzeugenberichten zufolge begann das Künstlerfest mit einer Art Sternmarsch, bei dem aus verschiedenen Himmelsrichtungen Wandergruppen zum Festplatz zogen. Es folgten Vorführungen, von denen besonders der künstlerische Tanz von Clotilde van Derp hervorgehoben wurde. Als es schon Abend wurde, fuhren die Teilnehmer auf Flößen Saale-abwärts davon, wobei die verschiedenen Gruppen sich in einem Sängerwettstreit zu überbieten trachteten. Nur wenige beschlossen den Tag mit einer gemeinsamen Übernachtung auf der Rudelsburg, darunter aber Carnap und etwa ein Dutzend Sera-Kreis-Mitglieder beiderlei Geschlechts. ${ }^{15}$ An dem Fest nahmen auch Studenten und Mitglieder des Lehrpersonals der Kunstgewerbeschule in Weimar teil, der Vorgängerinstitution des Bauhauses. Obwohl der Sera-Kreis offenbar eine unsichtbare Mauer um sich zog, dürfte seinen Mitgliedern klar geworden sein, dass man durchaus eines Geistes war. Das Ereignis wird Carnap und seinen Freunden deswegen schon die geistige Nähe der aktuellen Tendenzen in Philosophie und Wissenschaft mit denen im Neuen Bauen und im Design gezeigt haben und etwaige spätere Berührungsängste gar nicht erst aufkommen lassen haben.

Der Organisator des Festes, Eugen Diederichs, blickte nach dem Ende des Ersten Weltkriegs auf der ersten Friedens-Sonnenwendfeier des Kreises auf die Erlebnisse des Vorkriegsjahres 1913 zurück, die wohl die Höhepunkte des gesamten Vereinslebens markieren:

Und zuletzt als Krone unserer Feste 1913 das gemeinsame Fest mit der Weimarer Kunstschule, das große Werkbundfest unterhalb der Rudelsburg mit seinen 1200 Teilnehmern und der Tanzkönigin, der göttlichen Clotilde. [...] Sera hatte sich entwickelt zu einem „Jena und Weimar", es war ein Bund der Jenaer Universität und der Weimarer Kunstschule durch gemeinsame Erlebnisse junger Menschen geworden. ${ }^{16}$

Carnaps Freund Franz Roh, übrigens ebenfalls aus einer TextilfabrikantenFamilie (in diesem Fall aus dem thüringischen Apolda) stammend, hatte ursprünglich Germanistik (und Philosophie) studieren wollen. Er hat sich offenbar vor allem unter dem Einfluss von Herman Nohl, einem in Jena tätigen Philosophie-Dozenten, Dilthey-Schüler und geistigen Anführer des Sera-Kreises (Carnap 1963; Flitner 1986), später Pädagogik-Professor in Göttingen (Ratzke 1987), dazu entschlossen,

\footnotetext{
${ }^{15}$ wie Anm. 14.

${ }^{16}$ Eugen Diederichs: „Gedächtnisrede“, in: „Friedenssonnenwende ...“ (1919). Zum Zug auf den Hohen Meißner, dem anderen prägenden Ereignis des Kreises in diesem Jahr, setzte er fort:
}

Herbst 1913 zogen wir mit unserer soeben fertig gewordenen Fahne zum Hohen Meißner und weihten sie damit ein. So gehörte Sera mit zu dem gründenden Kreis der großen freideutschen Jugendbewegung. 
sich der Kunstgeschichte zuzuwenden (Flitner 1986, S. 126 f.; Werner 2003, S. 297). So studierte er zunächst in Basel bei Ernst Heidrich. Nach dessen Soldatentod schon in den ersten Kriegstagen im November 1914 setzte Roh seine Ausbildung in München bei Heinrich Wölfflin fort. Nach seiner Promotion entwickelte sich Roh schnell zu einem engagiertesten Verfechter der modernen Kunst in der Weimarer Republik.

Die Verbindung zwischen Carnap und den Freunden, insbesondere mit Roh, blieb in der Zeit des Krieges bestehen und wurde danach mit vielen gegenseitigen Besuchen bis zur Emigration Carnaps in der Mitte der 1930er-Jahre in die USA immer wieder erneuert. Bei der ersten Reise Carnaps in sein früheres Heimatland 1964 trafen sie sich ein letztes Mal im österreichischen Alpbach, nicht lange vor dem Tod Rohs am 30. Dezember 1965.

\subsection{Carnap und die Neue Sachlichkeit}

Während die Kontakte zwischen Carnap, Flitner und Roh in den Jahren des Ersten Weltkriegs nur durch Feldpostbriefe - und gelegentliche Treffen zwischen Carnap und Flitner hinter der Westfront - aufrechterhalten werden konnten, ${ }^{17}$ intensivierten sie sich danach wieder. Carnap lud nach seinem Umzug in sein neues Domizil in Buchenbach bei Freiburg mehr als einmal zu Diskussionen ein, etwa über Themen wie das System der Wissenschaften (Dahms 2016). Das scheint ein Ausgangspunkt zu seinem Logischen Aufbau der Welt gewesen zu sein, bei dem er die Freunde nicht ganz uneigennützig als intellektuelle Sparringspartner benutzte. Offenbar scheint er aber mit deren mangelndem Hintergrund in Logik und Erkenntnistheorie auf die Dauer nicht zufrieden gewesen zu sein. Deswegen suchte er sich - beginnend mit der Erlanger Konferenz von 1923 (Thiel 1993) - neue Gesprächspartner, die - wie Hans Reichenbach und Kurt Lewin - übrigens meistens ebenfalls aus dem Umkreis der Jugendbewegung vor dem Ersten Weltkrieg stammten.

Währenddessen profilierte sich Roh, der inzwischen bei Wölfflin über holländische Landschaftsmalerei promoviert worden war (Roh 1921), zum anfänglichen Missbehagen seines Doktorvaters als Kritiker und Chronist der aktuellen MalereiSzene (und zwar nicht nur der Weimarer Republik, sondern auch des Auslands). In München hielt er engen Kontakt zu den Ausstellungshäusern, die sich für moderne Kunst interessierten (Roh 1962, S. 95), nämlich Caspari, Goltz und Thannhauser, und lernte auf deren Veranstaltungen und Ausstellungen auch eine Vielzahl von modernen Künstlern persönlich kennen. Daraus gingen oft Besprechungen hervor, die in Zeitschriften wie dem Cicerone oder dem Kunstblatt gut nachzuverfolgen sind.

\footnotetext{
${ }^{17}$ Carnap-Tagebücher vom Mai bis August 1916, passim; zu den zahlreichen Themen, die die beiden bei ihren Treffen in der Nähe von Verdun diskutierten, gehörte insbesondere die Goethesche Farbenlehre, die sich Carnap hatte zusenden lassen. Siehe zu diesen Kontakten auch Flitner (1986, S. 206).
} 


\subsubsection{Die Mannheimer Ausstellung „Die Neue Sachlichkeit““ von 1925}

Eine dieser Ausstellungen ist besonders erwähnenswert, weil dort eine Münchener Künstlergruppe vorgestellt werden sollte, die aus Georg Schrimpf, Alexander Kanoldt, Carlo Mense und anderen Künstlern des später so genannten rechten Flügels der Neuen Sachlichkeit bestand (Georg Grosz vom später so genannten linken Flügel war allerdings auch bereits vertreten). Die Werke dieser Münchener Künstler zeigen häufig Landschaften, Architektur, Stillleben, Porträts etc., vermeiden aber meist die Schattenseiten des täglichen Lebens, vor allem in der Großstadt mit dem ganzen Elend der Nachkriegszeit des Ersten Weltkriegs - mit Kriegskrüppeln, Bettlern, Prostituierten etc. einerseits und einer herrschenden Klasse aus Kapitalisten, Juristen und Pfaffen andererseits -, ein Szenario, das vom so genannten linken Flügel der Neuen Sachlichkeit in seinen Gemälden sehr wohl beschrieben und angeprangert wurde.

Angesichts von Rohs Expertise ist es nicht weiter verwunderlich, dass der Mannheimer Museumsdirektor Gustav Hartlaub 1925 Roh kontaktierte, als er daran ging, die von ihm schon seit 1923 geplante, aber wegen der Nachkriegswirren verschobene, Ausstellung „Neue Sachlichkeit“ nun endlich in die Tat umzusetzen. Diese Zusammenarbeit hatte sich schon vorher bei einer Ausstellung von Bildern des Münchener Malers Ernst Haider in Mannheim bewährt. Wie der Briefwechsel zwischen Hartlaub und Roh zeigt, half Roh nicht nur mit einer Liste von neusachlichen Künstlern, wie er bescheiden schrieb, ${ }^{18}$ sondern auch mit Fotos von deren Werken und mit der Anbahnung von Kontakten zu den Künstlern, von denen Hartlaub nur wenige kannte.

Hartlaub schrieb u. a. an Roh:

Sehr geehrter Herr Dr. Roh!

Im Begriffe eine lange Auslandsreise anzutreten, möchte ich Ihnen noch schnell sagen, dass ich bei meinem letzten Aufenthalt im München - Sie waren leider noch abwesend - die Ausstellung nachexpressionistischer Malerei mit verschiedenen Künstlern, die schon durch Sie unterrichtet waren, besprochen habe. Es wurde vereinbart, die Ausstellung in diesem Sommer und zwar mit dem Eröffnungstermin 7. Juni zu veranstalten. Entsprechende Rundschreiben versende ich jetzt und werde nach meiner Rückkehr noch durch einige Reisen persönlich auswählen. Sie möchte ich noch herzlich bitten, uns in München bei der Zusammenstellung des Materials durch Beratung der Künstler etc. behilflich zu sein. Ich werde selbstverständlich im Katalogvorwort darauf hinweisen, dass die Idee dieser Ausstellung von uns gemeinsam gefasst worden ist und dass Sie uns bei der Zusammenstellung der Münchner Materials entscheidende Dienste geleistet haben.

Hoffentlich geht es Ihnen gesundheitlich wieder normal

Mit freundlichen Grüßen bin ich

\footnotetext{
${ }^{18}$ Siehe seine Erinnerung an diesen Sachverhalt in Roh (1925, S. 134). Sein Anteil an der Vorbereitung der Ausstellung wird dort heruntergespielt.
} 
Ihr ergebener

H. ${ }^{19}$

Hartlaub hat nur einen kurzen Einladungstext zur Ausstellung verfasst, in dem er das Auftauchen einer neuen Strömung gegenständlicher Kunst (nach Impressionismus und Expressionismus) thematisierte und auch deren verschiedene Unterarten unterschied, wie die eher neoklassizistische und von italienischen Vorbildern (wie den Valori Plastici um Carrá und de Chirico) und häufigen Aufenthalten dort geprägte Richtung der Münchener (von ihm der ,rechte Flügel“" genannt) und die Veristen um Grosz, Dix, Scholz und Hubbuch (von Hartlaub der „linke Flügel“ genannt).

In seinen Einladungsbriefen an die Künstler und Museumsleute liest sich das so:

Sehr geehrter Herr Rössing!

Schon lange plant die städtische Kunsthalle, in einer umfassenden Ausstellung diejenigen deutschen Maler zusammenzufassen, die nach Überwindung der expressionistischen Art zu einer kompositionell gebundenen, zugleich aber doch wieder gegenständlichen Darstellungsweise streben. Dabei kommen sowohl die mehr ,veristisch“ gerichteten, als auch die mehr im idealen Sinne gestaltenden Künstler in Frage. Nach Rücksprache mit einer Reihe von Malern ist beschlossen worden, die Ausstellung im Sommer ds. Js. stattfinden zu lassen. Die Eröffnung soll am 7. Juni stattfinden, die Ausstellung soll bis Mitte September dauern. Letzter Einsendungstermin ist der 25. Mai. ${ }^{20}$

\subsubsection{Rohs Buch Nach-Expressionismus und die Folgen}

Roh ging die Sache etwas anders und auch grundsätzlicher an: Er publizierte - wegen verzögert eingetroffener Fotos von Bildern einiger Maler leider erst nach dem Ende der Ausstellung - im Dezember 1925 sein Buch Nach-Expressionismus. Dieses Werk wurde sozusagen die Bibel der neuen Kunstrichtung. Zu seiner Verbreitung in dieser Funktion trug bei, dass es bei den weiteren Stationen der Ausstellung in Dresden, Chemnitz und schließlich in Dessau mit einer Binde versehen zum Verkauf auslag, auf der der einprägsame Titel der Mannheimer Ausstellung prangte: „Neue Sachlichkeit“.

Der Text fasste die neueste Kunstentwicklung zunächst weiter, wenn Roh - seinem Selbstverständnis eines Chronisten und Begleiters der neuen Kunst entsprechend - auch die Abstrakten und Konstruktivisten sowie etwa Max Beckmann als einen nicht eindeutig einem Flügel der Neuen Sachlichkeit zuordenbaren Künstler einbezog. Die französischen und spanischen Surrealisten werden dort im laufenden Text zwar immer wieder erwähnt, aber nicht in einem eigenen Kapitel thematisiert.

\footnotetext{
${ }^{19}$ Hartlaub an Roh, 27. März 1925 (Durchschlag), in: Archiv der Kunsthalle Mannheim.

${ }^{20}$ Hartlaub an Karl Rössing (Kunstgewerbeschule Essen), o. D., in: Archiv der Kunsthalle Mannheim.
} 
Das liegt allerdings nicht daran, dass Roh sie wegen eines auf die eigene Nation eingeschränkten Blickwinkels hätte links liegen lassen. Im Gegenteil wies er in seinem Buch immer wieder auf parallele Entwicklungen in Europa hin: Viele der von ihm aufgelisteten Maler sind aus Ländern außerhalb des europäischen Auslands.

Von besonderem Interesse ist das letzte Kapitel von Rohs Buch, in dem er auf im Gange befindliche „Umstellungen auf anderen Gebieten“ (wie der Architektur, der Literatur, der Musik etc.) hinwies bzw. diese mit seinen Bemerkungen manchmal sogar erst anstieß. Er erwähnte dort das Bauhaus für die Architektur, Franz Werfel, Carl Sternheim und Heinrich Mann für die Literatur, Arnold Schönberg (in seiner nachexpressionistischen Phase) und Igor Strawinsky in der ernsten sowie den Jazz in der populären Musik.

Am Ende des Kapitels findet sich ein - allerdings weniger detailliert ausgearbeiteter - Abschnitt über Wissenschaft und Philosophie. Roh beginnt diesen Abschnitt mit der ja ganz unbestreitbaren Feststellung: „Mit dem Expressionismus war auch Wissenschaftsverachtung verbunden gewesen“, und setzt etwas mysteriös fort: „Oder man hatte - gerade unter der Jugend - einer Scholastik oder einer Mystik im scheinwissenschaftlichen Sinne angehangen“ (Roh 1925, S. 114). Hier zeichne sich nun ein Wandel ab:

Auf eine geschlossene Reihe von Schriften gegen die Wissenschaft beginnen sich - unter der gleichen Jugend - wieder Verteidiger hervorzuwagen [...] Man kann hier beinah eine neue „Aufklärung“ konstatieren. Ebenso hat der gesamte Maschinenkult, wie er gerade unter den Künstlern herrscht, mindestens eine rationale Seite, so sehr er auch anders verstanden werden kann. (Roh 1925, S. 114)

Es fällt auf, dass Roh nicht nur eine Spaltung der Jugend konstatiert, sondern auch eine Spaltung zwischen der älteren Generation und eben dieser Jugend, wenn es um die Bewertung maßgeblicher Tendenzen des nicht lange zurückliegenden 19. Jahrhunderts geht:

[während] die Älteren beim 19. Jahrhundert dessen Rationalismus und Technizismus bekämpfen, die Jungen im 19. Jahrhundert aber gerade die Romantik, die Sentimentalität, den Triebirrationalismus samt seiner „Lebensphilosophie“, das unutopistische oder unkonstruktive Empfinden hassen. (Roh 1925, S. 115)

Roh nennt hier, anders als in den oben genannten Abschnitten, nicht Ross und Reiter. Ohne Kenntnis des geistigen und organisatorischen Zusammenhanges, in dem er selbst in der Jugendbewegung gestanden hatte, sind seine Bemerkungen kaum zu entschlüsseln. Wenn man diesen Kontext einbezieht, lässt eine Absage an die Lebensphilosophie hier jedenfalls aufhorchen. Denn diese hatte in Rohs geistiger Umgebung (wie dem Jenaer Sera-Kreis) wegen des großen Einflusses von Herman Nohl eine besondere Rolle gespielt. Wie Rohs Schriftwechsel mit Wilhelm Flitner zeigt, der nach wie vor im Banne der Lebensphilosophie stand, wird sein Bestreben deutlich, ihn von dieser Weltanschauung abzubringen und zur rationaleren Weltauffassung Rudolf Carnaps hinzuführen. 
Eine wichtige Stelle in Rohs Abschnitt über Wissenschaft und Philosophie ist einerseits auf den ersten Blick etwas rätselhaft, andererseits bei Einbeziehung des Hintergrundes doch wieder bezeichnend:

Es ist möglich, daß die neue Kunst sogar in unterirdischem Zusammenhange mit der neuesten Physik steht, nämlich mit ihrer Grundlage der ,starren Vierdimensionalität“, mit der diese neue Physik alles Dynamische ausrottet und alle Geschehen in Zustände zerlegen will. (Roh 1925, S. 116)

Wahrscheinlich verdankt sich diese Bemerkung Diskussionen, die Roh mit Rudolf Carnap während der Abfassung von Nach-Expressionismus in Carnaps Haus in Buchenbach bei Freiburg hatte. Carnap kannte sich ja nicht nur gut in der neuesten Physik aus, sondern hatte in seiner 1921 in Jena eingereichten Dissertation „Der Raum“ (Carnap 1922) auch Probleme der Relativitätstheorie behandelt.

Es ist insofern nicht erstaunlich, dass Carnap aufgefordert wurde, zur Klärung beizutragen, als Rohs erst wenige Tage vorher erschienenes Buch im Kreise von Freunden und Bekannten bei einem Skiurlaub in der Nähe von Davos beim Jahreswechsel 1925/226 auszugsweise vorgelesen und diskutiert wurde. ${ }^{21} \mathrm{Zu}$ dieser Runde gehörte Siegfried Giedion, der Architekturhistoriker, -theoretiker und -organisator, und László Moholy-Nagy, der konstruktivistische Maler und Kunsttheoretiker, der 1923 am Bauhaus die Durchführung des obligatorischen Vorkurses übernommen hatte. Davon schreibt Carnap, als er sich bei Roh nach der Rückkehr aus diesen Ferien für die Übersendung des Buchs bedankt:

Lieber Franz!

Endlich soll Dir für dein Buch Dank gesagt werden. Oft hab ich mir schon die Bilder angesehen, auch verschiedene Kapitel gelesen, freilich sehr mit Auswahl, immerhin in einem größeren Bruchteil, als Du später meiner „Konstitutionstheorie“ die Ehre geben wirst. In Glaris haben wir auch mal zusammen ein Kapitel vorgelesen; da kam der Vergleich mit Einstein vor; darüber sollte ich dann immer mal referieren, wir kamen aber nicht mehr dazu. ${ }^{22}$

Die erwähnte angebliche Analogie zwischen der neuesten Physik und der neusachlichen Malerei konnte damals also nicht geklärt werden. Sie hat die Anwesenden aber später noch ausführlich beschäftigt. Die Diskussion dürfte einer der Gründe gewesen sein, warum Giedion sein erst viel später erschienenes, aber wesentlich früher begonnenes berühmtes architekturtheoretisches Hauptwerk Space, Time and Architecture (Giedion 1941) schon im Titel in den Zusammenhang der neuen Physik gestellt hat.

\footnotetext{
${ }^{21}$ Auch im folgenden Jahr traf sich Carnap mit Moholy-Nagy und dessen Frau Lucia, der bekannten Fotografin, zum Skifahren und zu ausführlichen abendlichen Gesprächen; siehe das Carnap-Tagebuch vom 30. Januar $1927 \mathrm{ff}$.

${ }^{22}$ Carnap (Buchenbach) an Roh, 2. Februar 1926. Mit „Konstitutionstheorie“ ist übrigens Carnaps in Wien eingereichte Habilitationsschrift gemeint, die dann 1928 als Der logische Aufbau der Welt erschien. Moritz Schlick, der Gründer des Wiener Kreises, hatte Carnap von dem zu akademisch klingenden und zudem eventuell zu Verwechslungen mit Konstitutionstheorien in der Psychologie führenden Titel abgeraten.
} 
Darauf von Erich Mendelsohn angeschrieben, den Giedion stets nach Kräften aus der internationalen Gemeinde moderner Architekten herausgehalten hatte, hat Albert Einstein übrigens nur mit einem Spottgedicht geantwortet:

Lieber Herr Mendelsohn:

Die mir eingesandte Stelle aus dem Buch über Space, Time and Architecture hat mich zu folgender Antwort angeregt:

Nicht schwer ist's Neues auszusagen

Wenn jeden Blödsinn man will wagen.

Doch selt'ner füget sich dabei

Dass Neues auch vernünftig sei!23

Ich erwähne diese Geschichte hier nicht wegen ihres anekdotischen Wertes, sondern weil sich dahinter ein grundsätzlicheres Problem verbirgt. Sobald sich nämlich Kulturschaffende aus ganz anderen Gebieten für (gelegentlich auch nur imaginierte) Parallelen ihres Schaffens mit komplizierten wissenschaftlichen und philosophischen Theorien zu interessieren beginnen, kommt es unweigerlich zu Verkürzungen, Übertreibungen und auch Verdrehungen und Missverständnissen.

Ich finde es erstaunlich, wie sich trotzdem immer wieder schöpferische Impulse aus solchen Begegnungen ergeben. Man denke etwa an den am 5. Dezember 2012 (im Alter von 104 Jahren!) gestorbenen brasilianischen Architekten Oscar Niemeyer, der öfters an prominenten Stellen seiner Veröffentlichungen die Parallelen zwischen den Kurven seiner heimatlichen Umgebung in Rio de Janeiro und dem gekrümmten Universum Einsteins betont hat:

Es ist nicht der rechte Winkel, der mich anzieht, noch die gerade Linie, hart, unflexibel, durch den Menschen geschaffen. Was mich anzieht, ist die freie und sinnliche Kurve, die Kurve, der ich in den Bergen meines Landes begegne, dem gewundenen Lauf seiner Flüsse, den Wellen des Meeres, dem Körper der geliebten Frau. Aus Kurven besteht das ganze Universum, das gekrümmte Universum Einsteins. (Niemeyer 1998, S. 9 und 2005, S. 339; meine Übersetzung)

Mit diesen Ideen im Hintergrund ist Niemeyer ja tatsächlich zu einer mehr geschwungenen architektonischen Formensprache aufgebrochen, die den Architekturkubismus des internationalen Neuen Bauens hinter sich ließ; man denke an die Bauten seiner Parkanlage in Pampulha, danach in größerem Maßstab in Brasília, in Niterói und in São Paulo und zuletzt noch im Jahre 2011 in Avilés (Asturien). ${ }^{24}$

\footnotetext{
${ }^{23}$ Siehe auch den Abdruck des Gedichts in Georgiadis (1989, S. 139).

${ }^{24}$ Es handelt sich dabei allerdings durchweg um einzeln stehende Erholungs-, Repräsentationsund Regierungsgebäude. Auffällig ist, dass die Bürobauten für die meisten Ministerien in Brasília dem rechten Winkel und der geraden Linie folgen. Die Hunderte von Schulen, die Niemeyer konzipiert hat, sind - wegen der angestrebten Erweiterbarkeit - nicht nur Beispiele des rechtwinkligen Baukubismus, sondern sogar für den Plattenbau.
} 
Gehen wir wieder zu Carnap und seinem Interesse für moderne Kunst und besonders Architektur zurück! Er behielt dieses Interesse nach der Begegnung mit Giedion und Moholy-Nagy nicht nur bei, sondern intensivierte es noch. So besuchte er etwa die Internationale Ausstellung für Gesundheit, soziale Fürsorge und Leibesübungen („Gesolei““) im Sommer 1926 in Düsseldorf. ${ }^{25}$ Die Ausstellung im österreichischen Pavillon hatte dabei Otto Neurath ausgestattet. An deren Rand hatte er den Maler und Graphiker Gerd Arntz kennengelernt, der bei der Erfindung der Neurathschen Bildstatistik nach Wiener Methode eine entscheidende Rolle spielen sollte. Im folgenden Jahr besuchte Carnap die Stuttgarter Weißenhofsiedlung des Deutschen Werkbundes von 1927. Bei dieser bahnbrechenden Ausstellung moderner Wohnbau-Architektur war alles zugegen, was in Europa im Neuen Bauen Rang und Namen hatte. ${ }^{26}$ Carnap schrieb an Roh, dass er nach Stuttgart kommen wolle, „,sowohl um Euch zu treffen, als um der Ausstellung willen, die mir sehr am Herzen liegt“", ${ }^{27}$ hat dann aber offenbar nur eine Woche vor Schluss der Ausstellung (am 23. Oktober) zusammen mit seiner Frau Elisabeth die Häuserkolonie besuchen können, ohne dort aber die Rohs zu treffen, ${ }^{28}$ die schon früher dorthin gefahren waren. Carnaps Tagebuch enthält den folgenden Eintrag:

Sa 15 [1927, Verf.] Zur Siedlung „Weißenhof“, die Bauten der Werkbundausstellung. Das große Haus von Mies van der Rohe mit vielen Einzelwohnungen gefällt uns gut. Corbusier entsetzt Elisabeth wegen der Form und der trüben Farben. ${ }^{29}$

Im Jahr danach machte er in einer Passage des Vorworts zum Logischen Aufbau der Welt sein Interesse am Neuen Bauen auch öffentlich, als er Parallelen zur wissenschaftlichen Weltauffassung zog. Damit outete er sich damit in den Augen der Gegner solch progressiver Tendenzen als „Kulturbolschewist“. Sein Engagement zeigte sich dann weiter bei seinen Vorträgen am Bauhaus im Oktober 1929 in der Ära des (mittleren - nach Gropius und vor van der Rohe) Schweizer BauhausDirektors Hannes Meyer, über die ich - nach einer ersten Veröffentlichung von Peter Galison zu diesem Thema - an anderer Stelle berichtet habe. ${ }^{30}$ Dabei habe ich die These vertreten, dass der Wiener Kreis in der Ära Meyer der wichtigste Bezugspunkt der Bauhäusler in der Philosophie gewesen ist. Da diese These neuerdings von Peter Bernhard (2015) bestritten worden ist, sind hier ein paar Klarstellungen am Platze. Bernhard deutet etwa die Abwesenheit Meyers bei den ersten Vorträgen Carnaps als ein Zeichen von Desinteresse, wenn er schreibt: „Es lässt sich noch

\footnotetext{
${ }^{25}$ Carnap-Tagebuch vom 24. September 1926.

${ }^{26}$ Siehe dazu Kirsch (1987).

${ }^{27}$ Carnap an Roh, 6. September 1927.

${ }^{28}$ Das Bedauern darüber kommt zum Ausdruck in: Carnap an Roh, 20. Oktober und 08. November 1927.

${ }^{29}$ Aus dieser Bemerkung wird nicht deutlich, welches der beiden Häuser Corbusiers auf der Ausstellung gemeint war. Siehe für eine ausführliche Beschreibung Kirsch (1987, S. 112-130).

${ }^{30}$ Siehe Dahms (2004) mit einer vollständigen Liste der von Wiener-Kreis-Mitgliedern in der Ära Meyer am Bauhaus gehaltenen Vorträge und der Kommentierung einiger davon. Vgl. davor Galison (1990) und ausführlicher Bernhard (2021) in diesem Band.
} 
nicht einmal eine besondere Präferenz für diese Richtung [den Neopositivismus, Verf.] ausmachen " (Bernhard, 167). Das ist irreführend: Meyer war in diesen Tagen beim ersten öffentlichen Kongress der CIAM in Frankfurt am Main, bei dem das Thema „Die Wohnung für das Existenzminimum“ verhandelt wurde, das ihm sehr am Herzen lag. ${ }^{31}$ Der Umstand, dass in der Ära Meyer auch einige wenige andere Philosophen am Bauhaus vorgetragen haben, kann die These ebenfalls nicht entkräften: Aus Meyers Briefen an Josef Frank (und Otto Neurath) einerseits und an den Leipziger Karlfried von Dürckheim andererseits wird klar, dass er sich dem Wiener Kreis geistig näher stehend fühlte als etwa den Angehörigen der Leipziger Schule um Felix Krüger und Hans Freyer, ${ }^{32}$ die dann anschließend unter der Direktorenschaft Mies van der Rohes das wissenschaftlich-philosophische Begleitprogramm mehrheitlich bestimmten. ${ }^{33}$

Später in den USA hielt Carnaps Begeisterung für die Kulturmoderne und die Abneigung gegen rückwärts gewandte geistige und vor allem auch architektonische Strömungen an. Zwar kam es im Herbst1935 aus finanziellen Gründen nicht zu einer von Carnap angeregten Vortragsreise in die USA, wo er offenbar auch Vorlesungen über „,contemporary philosophy and culture“ hatte anbieten wollen. Die Informationen, die Ernest Nagel bei Sidney Hook darüber eingezogen hatte und an Carnap weiterleitete, sind bezeichnend, weil sie eine ähnliche politische und kulturelle Situation in den USA schildern, wie Carnap sie schon seit dem Ende der 1920er-Jahre in Mitteleuropa vorgefunden hatte:

Tell Carnap that universities throughout the U. S. are becoming politically more reactionary daily and to exclude from his prospectus anything which some dumb conservative - who ,feel' these things - might regard as cultural Bolshevism.

Deshalb riet Nagel ihm:

IN THE LIGHT OF THESE REMARKS. PERHAPS IT WOULD BE WISER IF YOU REPLACED THE LECTURE ON THE RELATION BETWEEN CONTEMPORARY PHILOSOPHY AND CULTURE BY SOMETHING LESS FULL OF DYNAMITE. ${ }^{34}$

Schließlich dann ein Jahr später doch glücklich in seinem Exilland angekommen, machte er sich in seiner „Intellectual Autobiography“ (Carnap 1963) über die neogotische Architektur auf dem Campus der University of Chicago lustig, die ihm zur metaphysischen Denkungsart seiner philosophischen Widersacher dort zu passen schien. ${ }^{35}$ Andererseits trug er zur Etablierung von Moholy-Nagys „New Bauhaus" in Chicago bei. ${ }^{36}$ Carnap mobilisierte auch Charles Morris, den Experten der Semiotik und Mitherausgeber bei der International Encyclopedia of Unified Sci-

\footnotetext{
${ }^{31}$ Siehe zu diesem CIAM-Kongress Dahms (2003, S. 86-106).

${ }^{32}$ Vgl. die Briefe an Graf Dürckheim und Josef Frank in Meyer (1980, S. 75-77).

${ }^{33}$ Siehe dazu Dahms (2002).

${ }^{34}$ Nagel an Carnap, 05.01.1935 (RC 029-05-16); siehe dazu auch Limbeck-Lilienau (2010), S. $130 \mathrm{f}$.

${ }^{35}$ Carnap (1963, S. 42).

${ }^{36}$ Siehe Findeli (1995).
} 
ence, sowie weitere Kollegen von der University of Chicago wie Ralph Gerard und Carl Eckardt für eine Zusammenarbeit mit Moholy-Nagys „New Bauhaus“ (Findeli 1995, S. 49). Er selbst beteiligte sich an der Serie von Abendveranstaltungen im Frühjahrssemester und hielt in diesem Rahmen einen Vortrag mit dem Titel „The Task of Science“, in dem er die Thesen aus seinem Vortrag „Wissenschaft und Leben“ am Dessauer Bauhaus im Oktober 1929 variierte und mit Beispielen aus seiner neuen Umgebung anreicherte (Findeli 1995, S. 64). ${ }^{37}$

Carnap traf sich 1939 in Harvard auch mit Giedion, der ein Angebot MoholyNagys zur Mitarbeit in Chicago abgelehnt hatte, um dessen Pläne für ein Institut für vergleichende Kulturforschung zu besprechen. Aufgabe des Instituts sollte es sein, die von Giedion so genannte „Methodengleiche“" zu untersuchen, also bewusste und vor allem unbewusste synchrone Parallelen zwischen auseinanderliegenden Kulturund eben auch Wissenschafts- und Philosophiebereichen. ${ }^{38}$ Aus diesen Plänen ist, nicht zuletzt wegen des Ausbruchs des Zweiten Weltkriegs, nichts mehr geworden. Und danach zog es Giedion vor, wieder nach Europa zurück zu kehren. Das Projekt war inzwischen begraben.

Die Frage, die sich hier stellt, ist in der Tat die, wie Carnaps Philosophie sich in den Kontext der Kunst und Kultur seiner Zeit einordnet, ob und gegebenenfalls welche „Methodengleiche“ etwa zwischen Malerei, Architektur, Literatur, Musik einerseits und der Carnapschen Philosophie andererseits besteht. Denn mögen die geschilderten persönlichen Kontakte und Bezugspunkte zu wichtigen Repräsentanten der Neuen Sachlichkeit so eng gewesen sein, wie sie wollen: Gibt es auch inhaltliche Parallelen? Meine These ist die, dass, während Roh einer der „Erfinder“ der Neuen Sachlichkeit und ihr wichtigster Theoretiker ist und Giedion deren geistiger Sachwalter in der Architektur, Carnap die entsprechende Rolle in der Philosophie einnimmt.

\title{
4.4 Carnap und die Neue Sachlichkeit
}

Im oben erwähnten Vorwort zum Logischen Aufbau der Welt heißt es ja immerhin:

\begin{abstract}
Wir spüren eine innere Verwandtschaft der Haltung, die unserer philosophischen Arbeit zu Grunde liegt, mit der geistigen Haltung, die sich gegenwärtig auf ganz anderen Lebensgebieten auswirkt; wir spüren diese Haltung in Strömungen der Kunst, besonders der Architektur, und in den Bewegungen, die sich um eine sinnvolle Gestaltung des menschlichen Lebens bemühen: des persönlichen und gemeinschaftlichen Lebens, der Erziehung, der äußeren Ordnungen im Großen. Hier überall spüren wir dieselbe Grundhaltung, denselben Stil des Denkens und Schaffens. Es ist die Gesinnung, die überall auf Klarheit geht und doch dabei die nie ganz durchschaubare Verflechtung des Lebens anerkennt, die auf Sorgfalt in der Einzelgestaltung geht und zugleich auf Großlinigkeit im ganzen, auf Verbunden-
\end{abstract}

\footnotetext{
${ }^{37}$ Zum 4-seitigen Manuskript des Chicagoer Vortrags siehe RC-110-08-21.

${ }^{38}$ Giedion-Nachlass: Institute for Contemporary History, Project o. D. (1941?) Archiv-Nr. 43-S-8-6.
} 
heit der Menschen und zugleich auf freie Entfaltung des Einzelnen. Der Glaube, dass dieser Gesinnung die Zukunft gehört, trägt unsere Arbeit. (Carnap 1928, Vorwort XX)

Von dieser Passage und anderen Äußerungen des Wiener Kreises ausgehend soll versucht werden, einmal die wichtigsten Merkmale der Neuen Sachlichkeit zusammenzustellen.

\subsubsection{Charakteristiken der Neuen Sachlichkeit}

Herbert Feigl hat schon nach seinen Vorträgen am Bauhaus im Sommer 1929 - also noch vor Carnaps Vorträgen im Oktober des Jahres - einen ersten Versuch unternommen, einige Charakteristika der neuen Denkweise anzugeben. In einem enthusiastischen Brief an seinen Doktorvater Moritz Schlick werden die Gemeinsamkeiten zwischen der Lehre des Wiener Kreises und der Haltung des Bauhauses auf einen folgenden Nenner gebracht:

Der neue Geist der Architektur ist ja, wie schon Carnap oft hervorhob, dem der neuen Philosophie sehr verwandt: der Kampf gegen die überflüssigen Wesenheiten (sentimentalkitschige Zieraten), die Nüchternheit, Sachlichkeit, Geradlinigkeit, Zweckangepaßtheit des neuen Bauens ist ja ausgesprochen positivistisch. Charakteristisch übrigens, dass Wittgensteins Bauideen im idealen Programm wenigstens mit den neuen Dessauer Prinzipien übereinstimmen. - Auch will man dort den Künstler-Individualismus zugunsten der Kollektivarbeit am sozial-Notwendigen nach Möglichkeit weitgehend überwinden. (So wie es auch in unserem philosophischen Kreis Kollektivarbeit gibt!). Und so wie bei uns jeder eine Einzelwissenschaft beherrscht, muss dort jeder Architekt ein Handwerk können. (Feigl an Schlick, 29.07.1929)

Diese Zusammenstellung kann man um einige Merkmale erweitern. Dann erhält man etwa folgende Liste von Charakteristika am Schnittpunkt von Wiener Kreis und Neuer Sachlichkeit:

- Konzentration auf Funktion und Struktur,

- Vermeidung des Beiläufigen, Ornamentalen,

- Reduzierung bzw. Ausschaltung des Emotionalen: Antipsychologismus,

- Zurückdrängung des Individuellen, Betonung des Kollektivs,

- Anti-Traditionalismus und forcierter Gegenwartsbezug,

- Internationalismus.

Diese Prinzipien sind hier sozusagen in absteigendem Grade der Verbindlichkeit angeordnet. Außerdem ist daran zu denken, dass nicht alle diese Charakteristika jeweils gleichzeitig auf allen hier diskutierten Gebieten (Malerei, Architektur, Philosophie) auftreten, bzw. nicht in gleicher Intensität. Insofern bezeichnet der Terminus „Neue Sachlichkeit“ ein Gebilde, das man sich nicht durch genus proximum und differentia specifica (wie in der traditionellen Definitionslehre) abgegrenzt denken muss, sondern eher durch den Wittgensteinschen Begriff der Familienähnlichkeit. 


\section{Funktionalismus und Kampf gegen das Ornament}

Die beiden erstgenannten und wichtigsten Prinzipien sind schon älteren Datums. Man denke an Louis Sullivans Dictum „Form follows function“ und Adoph Loos' Ausspruch vom „Ornament als Verbrechen“. Es handelt sich also bei ihrem Auftauchen in den 1920er-Jahren nicht um neue Prinzipien, sondern um ihre Anwendung auf die Umstände dieser Jahre sowie um ihre Kombination, die das Profil der Neuen Sachlichkeit ausmachen. Inhaltlich sind sie sozusagen korrelativ: Was keine Funktion hat, steht als bloßes Ornament dem Zweck der Sache entgegen und ist deswegen zu vermeiden bzw. abzuschaffen. Es ist nützlich, sich noch einmal die klassischen Formulierungen zu vergegenwärtigen.

Zunächst die positive Seite: In dieser Hinsicht wird die Neue Sachlichkeit durch die Verpflichtung auf den Funktionalismus ausgezeichnet. Dabei ist zu beachten, dass damit mehr gemeint ist als nur die Ansicht, ein Artefakt wie z. B. ein Werk der Architektur müsse auch funktionellen Ansprüchen genügen. Das ist bei Bauten nämlich schon mehr oder weniger so durchweg der Fall, dass es geradezu schwerfällt, in der Architekturgeschichte Bauten nachzuweisen, bei denen darauf verzichtet bzw. sogar bewusst dagegen verstoßen wurde, irgendeine Funktion zu ermöglichen. ${ }^{39}$ Insofern ist es auch kein Zufall, wenn schon die erste überlieferte architekturtheoretische Schrift, Vitruvs De Architectura, die Funktion (utilitas) als eines von mehreren Kriterien bzw. Zielen des Bauens - neben der Haltbarkeit (firmitas) und der Schönheit (venustas) - hinstellt. ${ }^{40}$

Vom selbstverständlichen Imperativ, man müsse auch funktionelle Aspekte berücksichtigen, und zwar im ausgewogenen Verhältnis zu den beiden anderen Prinzipien, unterscheidet sich der Funktionalismus nun aber durch seinen viel weiter gehenden Anspruch, dass es entweder auf andere Ziele (wie z. B. die Schönheit) nicht ankomme bzw. dass diese schon per se erreicht seien, wenn nur die Funktion optimal erfüllt würde. Als erste klassische Formulierung eines Standpunkts aus dem 20sten Jahrhundert, der die Funktion in den Mittelpunkt der architekturtheoretischen Debatte gerückt hat, gilt die Devise „Form follows function“, die 1901 von dem amerikanischen Architekten Louis H. Sullivan, dem Lehrer des berühmteren Frank L. Wright, publiziert wurde. Sullivan schreibt zur Begründung:

Ob wir an den im Flug gleitenden Adler, die geöffnete Apfelblüte, das schwer sich abmühende Zugpferd, den majestätischen Schwan, die weit ihre Äste breitende Eiche, den Grund des sich windenden Stroms, die ziehenden Wolken oder die über allem strahlende Sonne denken: Immer folgt die Form der Funktion - und das ist das Gesetz. Wo die Funktion sich nicht ändert, ändert sich auch die Form nicht. Die Granitfelsen und die träumenden Hügel bleiben immer dieselben: der Blitz springt ins Leben, nimmt Gestalt an und stirbt in einem Augenblick. Es ist das Gesetz aller organischen und anorganischen, aller physischen und metaphysischen, aller menschlichen und übermenschlichen Dinge, aller echten Manifesta-

\footnotetext{
${ }^{39}$ Die casa pendente (,schiefes Haus“) aus der Renaissance im Parco dei Mostri von Bomarzo nördlich von Rom ist ein schönes und seltenes Beispiel dafür.

${ }^{40}$ Siehe dazu Kruft (1995, Kap. 1, und dort besonders S. 24 ff.).
} 
tionen des Kopfes, des Herzens und der Seele, dass das Leben in seinem Ausdruck erkennbar ist, dass die Form immer der Funktion folgt. Das ist Gesetz [... $]^{41}$

An dieser Äußerung ist - außer ihrem für den Autor charakteristischen poetischen Überschwang - eine Schwäche auffällig: Sullivan fasst den Funktionsbegriff so weit, dass darunter außer Teilen der belebten Natur (wie z. B. einzelnen Körperteilen und -organen) und Artefakten (wie den verschiedensten Gebrauchsgegenständen und insbesondere Werkzeugen und Maschinen), denen wir normalerweise eine Funktion zuerkennen, nicht nur belebte Ganzheiten (wie Adler, Zugpferd etc.) fallen, sondern sogar Teile der unbelebten Natur (wie Granitfelsen und träumende Hügel), bei denen wir üblicherweise nicht von einer Funktion sprechen würden.

Von dem viel zitierten funktionalistischen Prinzip Sullivans kann man spätere, aggressivere Formulierungen aus den 1920er-Jahren abheben, wie sie etwa in Hannes Meyers (des zweiten Bauhausdirektors) Devise „Funktion mal Ökonomie“ verkündet werden:

Unbelastet von klassischen Allüren, künstlerischer Begriffsverwirrung oder kunstgewerb-
lichem Einschlag erstehen [...] die Zeugen einer neuen Zeit. Muster-Messe, Getreide-Silo,
Music-Hall, Flug-Platz, Bureau-Stuhl, Standardware. Alle diese Dinge sind ein Produkt der
Formel: Funktion mal Ökonomie. Sie sind keine Kunstwerke. Kunst ist Komposition,
Zweck ist Funktion. (Meyer 1989 [1926], S. 71)

Die Formel „Funktion mal Ökonomie“ ist hier vielleicht erläuterungsbedürftig. Man fragt sich, warum zur Funktion anders als bei Sullivan noch Ökonomie tritt. Die Idee dahinter ist offenbar zunächst einmal, dass Funktion allein nicht ausreicht. Wäre es so, könnte man einen optimal funktionierenden Gegenstand beliebig teuer machen. Die Kosten setzen dem Nutzen des Gegenstandes jedoch Grenzen. - So viel zum „Funktionalismus“ als positivem Kernpunkt der Neuen Sachlichkeit.

Als Großvater der „Neuen Sachlichkeit“ in der Architektur Europas wird gern der Wiener Adolf Loos genannt. Von ihm kann man besonders gut den negativen Kernpunkt in der Programmatik der Neuen Sachlichkeit herleiten, in den ebenfalls ökonomisches Denken hineinspielt. Ich meine seine wohlbekannte Losung vom „Ornament als Verbrechen“. Ich zitiere seine Äußerungen im Kontext etwas ausführlicher, weil er merkwürdigerweise sowohl für Anhänger als auch für Kritiker der Sachlichkeit eine entscheidende Bezugsperson gewesen ist.

Loos schreibt also im Jahre 1908 folgende Philippika: ${ }^{42}$

Der ungeheure Schaden und die Verwüstungen, die die Neuerweckung des Ornamentes in der ästhetischen Entwicklung anrichtet, könnten leicht verschmerzt werden, denn niemand, auch keine Staatsgewalt, kann die Evolution der Menschheit aufhalten. Man kann sie nur verzögern. Wir können warten. Aber es ist ein Verbrechen an der Volkswirtschaft, dass dadurch menschliche Arbeit, Geld und Material zugrunde gerichtet werden. Diesen Schaden kann die Zeit nicht ausgleichen.

\footnotetext{
${ }^{41}$ Zitiert nach der deutschen Übersetzung in Albert (1968, ???); siehe dazu auch Kruft (1995, S. $410 \mathrm{ff}$.).

${ }^{42} \mathrm{Zu}$ Loos und seiner Haltung zum Ornament siehe Kruft (1995, S. 419-422 und die dort 634 angegebene Literatur).
} 
Die Nachzügler verlangsamen die kulturelle Entwicklung der Völker und der Menschheit, denn das Ornament wird nicht nur von Verbrechern erzeugt, es begeht ein Verbrechen dadurch, dass es den Menschen schwer an der Gesundheit, am Nationalvermögen und also in seiner kulturellen Entwicklung schädigt. (Loos 1982 [1908])

Loos argumentiert hier, ganz anders als noch Sullivan, der das Vorbild der unverbildeten Natur für seine Ansicht ins Feld führte, hauptsächlich mit ökonomischen Notwendigkeiten, aus denen seiner Ansicht nach offenbar kulturelle Entwicklungen folgen sollen. Sowohl historisch als auch systematisch gesehen ist diese Berufung auf Sachzwänge aber problematisch. Die größten Kunstwerke und architektonischen Meisterwerke der Geschichte hätte es bei einem Beharren auf einer ökonomistischen Perspektive nicht gegeben. Auch hinsichtlich gegenwärtiger Architektur ist die ökonomische Perspektive nicht immer die beste Lösung. Denn ökonomische Sparsamkeit generiert nicht immer das unter Funktionsgesichtspunkten sozusagen kulturell Optimale. Außerdem ist Loos - man könnte sagen: zu seinem Glück nicht immer konsequent gewesen: Er hat in seinen eigenen Schöpfungen durchaus Elemente verwendet, die jedenfalls nicht funktional bedingt sind und insofern selbst aus heutiger Sicht als ornamental bezeichnet werden könnten (wie etwa sein berühmtes Haus am Michaelerplatz gegenüber der Wiener Hofburg von $1911^{43}$ oder erst recht sein späterer - dann aber nicht realisierter - Entwurf für den Neubau der "Chicago Sun“44).

Diese beiden Charakteristika, der Funktionalismus einerseits und die Kritik am „Ornament" andererseits, machen also den Kern der Neuen Sachlichkeit aus.

Wie die obigen Zitate zeigen, entstammt der Funktionalismus aus theoretischen Diskussionen und deren Anwendungen in der Architektur. Das ist kein Wunder, weil es sich bei dieser Disziplin um eine angewandte Kunst handelt, wo der Bezug auf den Gebrauch durch die Nutzer besonders naheliegt. In der Malerei und erst recht in der Philosophie mag es dagegen schwerer sein, funktionalistische Denkmuster zu entdecken.

Wie steht es damit in der Malerei? Hier hat Sigfried Giedion, der ja zusammen mit Roh Kunstgeschichte studiert hatte und sich noch bis in die 1930er-Jahre auch als Ausstellungsmacher am Züricher Kunsthaus engagierte, in einem Vortrag bei den Davoser Hochschulwochen im Jahre 1931 - zwei Jahre nach Martin Heideggers berühmtem Auftritt dort! - eine Ortsbestimmung versucht:

Dieses Besinnen auf die eigentlichen Aufgaben vollzieht sich auf den meisten Gebieten und findet wohl immer statt, wenn auf lange Sicht hinaus Grundsteine für ein neues Weltbild gelegt werden müssen. In der Literatur, in der Philosophie, in der Architektur wie in der Malerei, überall wird nach den Grundelementen gefragt, die jeder Disziplin eigen sind.

(Giedion 1931, S. 21)

Dabei sei es „,die Pflicht eines jeden, auf seinem Gebiet Ordnung zu schaffen“ und zu dem nötigen „Reinigungsprozeß“ beizutragen (Giedion 1931, S. 20). Dann

\footnotetext{
${ }^{43}$ Siehe dazu Czech und Mistelbauer (1984).

${ }^{44}$ Siehe dazu Loos (1983, linke Umschlagseite innen und S. 194 ff.).
} 
kommt Giedion unter der Überschrift „Die Funktion heutiger Malerei“ auf die bevorstehenden Aufgaben in dieser Kunstsparte zu sprechen:

Die Malerei, die beschreibend mit den in den letzten Jahrhunderten geschaffenen Mitteln und sicher mit äußerster Geschicklichkeit die Welt und die persönlichen Erlebnisse des Malers zur Abbildung bringt, befriedigt uns nicht mehr. Es interessiert uns nicht, darüber informiert zu werden, wie der Maler sein Haus, seine Welt, seinen Blumenstrauß, seine Maske oder seine Dämonie sieht. Die Mittel, mit denen dies geschieht, erscheinen verbraucht und im Widerspruch mit unserem inneren Aufbau. (Giedion 1931, S. 21)

Er schildert auch, wie er sich die Funktion der Malerei stattdessen vorstellt:

Die Malerei, von der wir reden wollen, will heute gar nicht mehr „peinture“ sein, wie auf anderen Gebieten will sie über ihr rein ästhetisches Dasein hinaus wirksam werden. Die psychologische Wiedergabe der Welt interessiert sie im Grund nicht. Sie schafft eine neue Realität, indem sie auf die Urelemente der Malerei selbst zurückgreift. Nur dadurch erscheint ein Reinigungsprozeß möglich. (Giedion 1931, S. 21)

Der Maler würde auf diese Weise zum „Seismographen kommender Entwicklung“. Zwar sei es weiterhin möglich und erwünscht, sich traditionellen Sujets wie der Landschaftsmalerei zu widmen. Dabei sei jedoch keine romantische Stimmungsmalerei mehr gefragt, sondern ganz neue Blickwinkel und Perspektiven seien gefordert, nämlich einerseits der weitere, zusammenfassende „Flugzeugblick“, andererseits der schärfere, ins winzige Detail gehende mikroskopische Blick.

Wie sieht es nun mit dem Funktionalismus und dem Kampf gegen das Ornamentale und Überflüssige in der Philosophie Carnaps aus, den Giedion in seinem Davoser Vortrag zusammen mit dem Wiener Kreis als Vorbild für die neue Entwicklung in der Philosophie nennt? Die Kritik am überflüssigen „Ornament“ und die Verwendung eines funktionalistischen Sprach- und Wissenschaftsverständnisses bei Carnap werden im Folgenden wegen ihrer argumentativen Interaktion in einem Atemzug beschrieben. Ein erster Beleg für ein funktionalistisches Philosophieverständnis zeigt sich bei Carnap in seinem Vortrag „,Der Mißbrauch der Sprache“ am Dessauer Bauhaus vom Oktober 1929. Dort bringt er eine funktionalistische Sprachtheorie zur Anwendung, die zwischen der deskriptiven, der appellativen und der emotiven Funktion der Sprache unterscheidet. Sie ist von dem Wiener Philosophen und Psychologen Karl Bühler entlehnt, in dessen Buch Die Axiomatik der Sprachwissenschaften sie dann 1933 erschien. ${ }^{45}$ Carnaps Anwendung dieser Theorie konzentriert sich im Dessauer Vortrag auf eine Kritik an Vermischungen solcher Sprachfunktionen, wenn also etwa deskriptive und emotive Funktion durcheinandergeraten. Dabei kommt er zu folgendem Resultat: Die Metaphysiker tun so, als würden sie (deskriptiv) etwas Sachhaltiges behaupten. In Wahrheit drücken sie aber (emotiv) nur Gefühle aus. Und das in einer schlechten Weise. Denn das solle man in der Kunst tun.

Ein berühmtes Beispiel für diese angebliche Funktionsverwirrung ist Carnaps Besprechung der Heideggerschen Metaphysik, wie sie nicht schon im Dessauer Vortrag, ${ }^{46}$ sondern erst in dem späteren - Elemente des Dessauer Vortrags nutzen-

\footnotetext{
${ }^{45}$ Siehe dazu auch Graumann und Herrmann 1984.

${ }^{46}$ Carnap „Mißbrauch der Sprache“ (RC 110-07-43:1)
} 
den - publizierten Aufsatz „Überwindung der Metaphysik durch logische Analyse der Sprache“ (Carnap 1932) vorgeführt wird. Der immer wieder gern zitierte Heideggersche Satz ist natürlich „Das Nichts selbst nichtet“ (Heidegger 1929, S. 34; dort werden auch solch hübsche Wortschöpfungen wie die „Nichtung“ [S. 34] und das [nicht: die] „Nichten“ [S. 35] vorgeführt) ist nach Carnaps Ansicht nicht einmal zur Evokation von Emotionen zu gebrauchen, sondern lediglich multipler syntaktischer Unsinn. Wenn man den Satz - sozusagen als Werkzeug - in die Alltagswelt mit ihren vielfältigen Gebrauchsgegenständen rückübertragen wollte, handelte es sich bei diesem Missbrauch des geistigen Werkzeugs Sprache sozusagen um so etwas wie den untauglichen Versuch, mit einem Gummischwamm einen Nagel in die Wand zu treiben.

Carnap greift also in negativer Hinsicht bei seiner Polemik gegen die Metaphysik auf eine funktionalistische Sprachtheorie zurück. Er benutzt sie aber auch in positiver, konstruktiver Weise, wenn er empfiehlt, Ethik und Ästhetik nicht deskriptiv - und deshalb mit einem Wahrheitsanspruch verbunden - zu verstehen, sondern emotivistisch aufzufassen, also ethische Wertungen und Normen als Ausdrücke von Emotionen. Diese von Carnap nur sehr skizzenhaft proklamierten Ideen sind dann in der Ethik von Alfred Ayer und L. E. Stevenson aufgegriffen und weiterentwickelt worden.

Weiterhin hat Carnap ein funktionalistisches Wissenschaftsverständnis, demzufolge wissenschaftliche Theorien Werkzeuge (und zwar in diesem Fall:) der Prognose sind. Das ist natürlich nichts ganz Neues; man findet ähnliche Ideen spätestens bei Auguste Comte (wenn nicht schon bei Francis Bacon), dann später bei den Pragmatisten, Wilhelm Ostwald und anderen.

Dieses Verständnis von Sprache und Wissenschaft war im Wiener Kreis nicht unumstritten. Es wurde von Carnaps Kollegen im linken Flügel des Wiener Kreises wie Otto Neurath und Philipp Frank geteilt. Neurath etwa hielt wissenschaftliche Theorien für intellektuelle Werkzeuge und nannte als deren Zweck die Vorhersage, ${ }^{47}$ geradezu „das Um und Auf der Wissenschaft“ (Neurath 1931a/1981, S. 418). Diese Sicht wurde dagegen von Moritz Schlick als Vertreter des rechten Flügels für das Verständnis abstrakter Theorien wie etwa solcher der theoretischen Physik zurückgewiesen. Er stritt nicht ab, dass Theorien aus praktischen Beweggründen entstanden sein mochten. Aber sie hätten sich von extrinsischen Zwecken (wie eben der Vorhersage) emanzipiert und seien seitdem intrinsischen Zwecken (wie der Wahrheitsfindung) verpflichtet.

So viel zu den Kernprinzipien der Neuen Sachlichkeit und ihrer Bedeutung in Malerei, Architektur und Philosophie.

\section{Anti-Psychologismus}

Das Thema wird in diesem Band ausführlicher schon in den Beiträgen von Uljana Feest und Thomas Uebel behandelt. Es geht um Carnaps Wendung vom Phänomenalismus zum Physikalismus, einer damals von Carnap und Neurath propagierten sozusagen aktualisierten Version des Materialismus. Dabei bleibt umstritten, ob

${ }^{47}$ So in Neurath (1931b/1981), S. 426, 514). 
Carnap auch jene von Otto Neurath behavioristische Wendung mitvollzogen hat, nach der in der Psychologie menschliche Handlungen auf äußerlich wahrnehmbare Körperbewegungen reduziert werden. Nicht der Beobachtung zugängliche metaphysikverdächtige Entitäten wie Gedanken und Emotionen werden demnach aus dem wissenschaftlichen Repertoire ausgeschlossen. Dinge wie „Einfühlung“, wie sie etwa in der Lebensphilosophie noch eine Rolle spielten, um Zugang zu den Emotionen von Personen zu erhalten, werden allenfalls als Behelfe angesehen. Sie dienen dem Forscher - nach einem Bonmot von Otto Neurath - bei seiner Arbeit nicht mehr und nicht weniger als „ein guter Kaffee“ (Neurath 1931b/1981, S. 463).

\section{Gegen Individualismus, für Kollektivismus}

Bei allen hier genannten Anhängern der Neuen Sachlichkeit (also Carnap, Giedion und Roh) fällt zunächst einmal auf, dass sie sich während ihres Berufslebens fast ständig im Rahmen von Gruppen bewegt haben, seien es nun der Kreis der Münchener Maler im Falle Rohs, die Internationale der modernen Architektur im Falle Giedions oder der Wiener Kreis und später in der Emigration in den USA die weitere Bewegung des Logischen Empirismus im Falle Carnap.

Aber wie weit geht der „Kollektivismus“ bei Carnap? Im Vorwort zu seinem Logischen Aufbau - wie auch in Feigls oben zitiertem Brief an Schlick - finden wir ein plakatives Bekenntnis zur kollektiven Zusammenarbeit:

Die Grundeinstellung und die Gedankengänge dieses Buches sind nicht Eigentum und Sache des Verfassers allein, sondern gehören einer bestimmten wissenschaftlichen Atmosphäre an, die ein Einzelner weder erzeugt hat, noch umfassen kann. Die hier niedergeschriebenen Gedanken fühlen sich getragen von einer Schicht von tätig oder aufnehmend Mitarbeitenden. Gemeinsam ist dieser Schicht vor allem eine gewisse wissenschaftliche Grundeinstellung (Carnap 1928, XVIII).

Viele philosophische Probleme wurden in der Tat bei regelmäßigen Treffen im Wiener Kreis oder an dessen Rande gemeinsam ausführlich, oft auch kontrovers, diskutiert, um erst dann publiziert zu werden. Es gab auch gemeinsame Publikationsanstrengungen wie die Zeitschrift Erkenntnis sowie die beiden Publikationsreihen des Kreises: die „Schriften zur wissenschaftlichen Weltauffassung“ und die Reihe „Einheitswissenschaft“. Für die Zeit der Emigration bzw. des Exils seit 1933/34 wäre noch die International Encyclopedia of Unified Science zu erwähnen. Jedoch: Außer bei der Programmschrift des Wiener Kreises ging der „Kollektivismus" nicht so weit, dass eine gemeinsame Autorenschaft propagiert oder praktiziert worden wäre.

Diesen gemeinsamen Anstrengungen wäre in negativer Hinsicht die Ablehnung der gegenteiligen Haltung gegenüberzustellen, d. h. eines Individualismus, wie er etwa in der Verherrlichung der großen Einzelnen zum Ausdruck kommt, also jener Herrschaften, die vermeintlich die politische, die kulturelle wie auch die wissenschaftliche Geschichte exklusiv bestimmt hätten. Dieses Konstrukt hat Edgar Zilsel (1990 [1918]) „Geniereligion“ genannt und entsprechend kritisiert.

\section{Antitraditionalismus und forcierter Gegenwartsbezug}

Hier nehme ich als Einstieg eine Passage aus dem Manifest des Wiener Kreises: 
Die Vertreter der wissenschaftlichen Weltauffassung stehen entschlossen auf dem Boden der einfachen menschlichen Erfahrung. Sie machen sich mit Vertrauen an die Arbeit, den metaphysischen und theologischen Schutt der Jahrtausende aus dem Weg zu räumen. (Verein Ernst Mach 1929/1981, S. 314)

Von verschiedenen Kritikern des Wiener Kreises ist nach Lektüre von Stellen wie dieser behauptet worden, dass dieser ein geradezu nihilistisches Verhältnis zur philosophischen Tradition gehabt habe. Man denke für ein besonders radikales Statement einer solchen Kritik nur an folgende Stelle aus Max Horkheimers Polemik gegen den Logischen Positivismus in seinem Aufsatz „Der neueste Angriff auf die Metaphysik“"von 1937:

Aristoteles und Kant und Hegel [gelten ihnen, Verf.] als die größten Wirrköpfe, ihre Philo-
sophie als ein wissenschaftliches Nichts, bloß weil sie nicht zur Logistik passt und die Be-
ziehung zu den „Wurzelbegriffen“ und „Elementarerlebnissen“ des Empirismus problema-
tisch ist. In der Oberflächlichkeit und Anmaßung, mit der hier über geistige Leistungen
geurteilt wird, kündigt sich ein Verhältnis zum kulturellen Erbe an, das sich praktisch zu-
weilen bei nationalen Erhebungen und ihren Freudenfeuern zu betätigen pflegt, mögen die-
sen jenen Autoren persönlich noch so zuwider sein. (Horkheimer 1937, S. 41)

Wenn man das Reden von „nationalen Erhebungen und ihren Freudenfeuern“ hier einmal in Klartext übersetzt, so wird den Positivisten in diesem Text aus dem Jahre 1937 also geradezu ein Verhältnis zur philosophischen Tradition vorgeworfen, wie es sich vier Jahre zuvor bei den nationalsozialistischen Bücherverbrennungen vom 10. Mai 1933 ausgetobt hatte.

Mir scheint Horkheimers Kritik - von der angeblichen Parallele zu den NaziAktivisten einmal abgesehen - für die 1920er und frühen 1930er-Jahre - man denke an die Rede vom „Schutt der Jahrtausende“ in der Programmschrift des Wiener Kreises - teilweise berechtigt. Der Wiener Kreis hat mit seiner internationalen Ausbreitung in der Mitte der dreißiger Jahre dann aber auch intensivere Anstrengungen unternommen, sich eine geistesgeschichtliche Genealogie zuzulegen. Man vergleiche dazu die entsprechenden - freilich zu recht unterschiedlichen Resultaten gelangenden - Bemühungen Neuraths, aber auch Schlicks, im Vorfeld des Pariser Internationalen Kongresses für Philosophie von 1937. Während Schlick die Essenz der wissenschaftlichen Philosophie, das Klären von Begriffen und Gedanken, schon auf die sokratische Technik in den platonischen Dialogen zurückführen wollte, legte Neurath Wert auf die Anknüpfung an die Tradition der Französischen Aufklärung, insbesondere an die Enzyklopädisten um Diderot und d'Alembert. ${ }^{48}$

Wie stand es mit dieser Thematik eines bewussten oder eben auch bewusst verweigerten Traditionsbezugs bei den Protagonisten der Neuen Sachlichkeit Franz Roh, Sigfried Giedion und Rudolf Carnap?

Bei den beiden Erstgenannten ist zunächst daran zu erinnern, dass es sich bei ihnen um Kunsthistoriker gehandelt hat, die bei ihrem Doktorvater Heinrich Wölfflin in München eine solide Ausbildung erhalten hatten und sich in den von ihm be-

\footnotetext{
${ }^{48}$ Siehe dazu Näheres in Dahms 1996.
} 
treuten Dissertationen noch mit ganz traditionellen historischen Themen beschäftigt hatten. ${ }^{49}$ Das wurde anders, als sie in ihren folgenden Büchern dann die Zeitgeschichte der Malerei und Architektur der unmittelbaren Gegenwart in den Blick nahmen. Diesen plötzlichen Wandel haben sie in ihren Büchern NachExpressionismus und Bauen in Frankreich auch explizit zur Sprache gebracht. So schreibt Roh direkt nach seiner Einleitung in dem kurzen Kapitel „Gegenwartsgeschichte überhaupt":

Aller geschichtlichen Befassung mit Gegenwartsproblemen haften Sonderschwierigkeiten an. Man pflegt aber den Historiker der „Gegenwart“ (d. h. hier immer nur der jüngsten Vergangenheit) mindestens von seiten der Zunft mit falschem Mißtrauen zu begrüßen: so nahen Verhältnisse könne man nur subjektiv gegenüberstehen.

Wohl wollen wir zugeben, dass die Verhältnisse aus Nahsicht heraus sich leicht verschieben, auch Haupt- und Nebenlinien schwerer aus dem Gewirr des Ganzen auseinandertreten. Dafür aber hat man bei der Gegenwart das volle Bild vor sich. Es steht lückenloser ausgebreitet da, als etwa eine entsprechende Generation des Mittelalters, wo erstens schon furchtbarster Materialausfall das Bild verzeichnet, zweitens die Unmöglichkeit hervortritt, sich restlos in den Geist entschwundener Zeitläufte zu versetzen. (Roh 1925, S. 5)

Roh betont auch, dass ,zu fordernde methodische Strenge“ auch gegenüber Objekten der Gegenwart eingehalten werden könne. Denn

wenn der Betrachter nämlich wirklich geschichtliche Bildung erwarb, ja historischen Instinkt besitzt, so hat er den auch gegenüber seiner eigenen Zeit. (Roh 1925, S. 6)

Wie er im folgenden Kapitel ,Verachtung unserer Zeit“" ausbreitet, fühlt er sich aber nicht nur als unparteiischer, sondern auch als positiv gestimmter Begleiter der Kunst der Gegenwart. Denn er erklärt dort, dass er für „,das Gewimmer vom künstlerischen Untergange Europas und der Zerrissenheit der Zeit" nichts übrig habe, wie es etwa in Oswald Spenglers Untergang des Abendlandes angestimmt wurde (Roh 1925, S. 7). ${ }^{50}$

Sigfried Giedion hat ebenfalls gleich auf der ersten Seite seines Buchs Bauen in Frankreich in den zwei Seiten „Zur Einleitung“ den Zeitbezug angesprochen: „Auch der Historiker steht in der Zeit, nicht über ihr. Das Ewigkeitspostament hat er verloren“ (Giedion 1928/2000, S. 1). Noch auf derselben Seite äußert er sich über die neue Rolle des Zeitgeschichtlers so:

Aufgabe des Historikers ist es, vorab die Keime zu erkennen, und - über alle Verschüttungen hinweg - die Kontinuität der Entwicklung aufzuzeigen. Leider benützte der Historiker den Überblick, den seine Beschäftigung mit sich brachte, um die ewige Berechtigung des Vergangenen zu verkünden, und die Zukunft damit totzuschlagen. Zumindest aber, um hemmend die Entwicklung aufzuhalten.

\footnotetext{
${ }^{49}$ Es handelt sich um Roh (1921) und Giedion (1922).

${ }^{50}$ Spengler wird an dieser Stelle allerdings nicht genannt. Er war ihm aber gewiss schon länger bekannt, nicht zuletzt aus Diskussionen mit Otto Neurath, der 1921 seinen Anti-Spengler publizierte (Neurath (1921)).
} 
Die Aufgabe des Historikers scheint uns heute die entgegengesetzte zu sein: Aus dem ungeheuren Komplex einer vergangenen Zeit jene Elemente herauszuschälen, die zum Ausgangspunkt der Zukunft werden. (Giedion 1928/2000, S. 1)

Wie steht es bei Carnap mit der Einschätzung des Verhältnisses von Geschichte und Gegenwart in der Philosophie? Wie schon eingangs erwähnt, hat bei ihm die Begegnung mit dem klassischen griechischen Bildungsgut schon früh während der Schulzeit und besonders durch die Reisen in der Obhut seines Onkels Wilhelm Dörpfeld eingesetzt. ${ }^{51}$ Danach hat er sich während seines Studiums dann schon eher als Roh und Giedion mit der Gegenwart seines Studienfachs auseinandergesetzt, insbesondere mit der mathematischen Logik Gottlob Freges und Bertrand Russells. Es ist insofern auch kein Wunder, dass er seine Dissertation Der Raum von 1921 keinem historischen Thema mehr gewidmet hat, sondern dem Versuch, die kantische Behandlung des Raumbegriffs den neuen Erkenntnissen der Physik, insbesondere der Einstein'schen Relativitätstheorie, gegenüberzustellen. Seine Habilitationsschrift Der logische Aufbau der Welt ist ganz der Aufgabe gewidmet, durch Anwendung der neuen mathematischen Logik eine neue Grundlage für die Erkenntnistheorie zu gewinnen.

Dabei hat Carnap - ebenso wie Roh und Giedion hinsichtlich der Malerei und Architektur - Anlass, sich vom traditionellen Rollenbild des Gelehrten in seiner Disziplin, der Philosophie, abzuwenden. Während er den Naturwissenschaften und auch den Historikern attestiert, ihre Arbeit als Teil einer arbeitsteilig betriebenen Gesamtwissenschaft aufzufassen (Carnap 1928, XIX f.), sei das bei den Philosophen anders: Sie seien bislang oft darauf aus gewesen, ,ein ganzes Gebäude der Philosophie in kühner Tat zu errichten“. Dabei habe ihr Zugang oft genug ,,mehr der eines Dichtenden“ geglichen. Mit dem Einzug eines arbeitsteiligen und vor allem argumentativ verstandenen Verständnisses der Philosophie ergebe sich nun die Aufgabe einer „Ausschaltung des spekulativen, dichterischen Arbeitens in der Philosophie" (Carnap 1928, XIX f.).

Offenbar hatte Carnap also den Eindruck, dass er es in der Philosophie mit einer schwierigeren Aufgabe zu tun habe als Roh und Giedion: Während in deren Disziplinen eine wissenschaftliche Einstellung und arbeitsteilige Vorgehensweise schon vorhanden sei und der zu überwindende Widerstand darin bestehe, diese Tendenzen nicht nur einer antiquarischen Kunstauffassung zugute kommen zu lassen, sondern der Aufarbeitung der Gegenwart nutzbar zu machen, hatte Carnap es in der Philosophie (nach seiner Auffassung) mit einem schwereren Problem zu tun: überhaupt erst wissenschaftliches Arbeiten und Arbeitsteilung einkehren zu lassen. Nichtsdestoweniger hat er im Aufbau noch immer wieder außer zahlreichen Beispielen auch Hinweise auf historische Literatur eingeschaltet.

\section{Internationalistische Ausrichtung}

Man kann darüber diskutieren, ob Internationalismus tatsächlich zu den Merkmalen der Neuen Sachlichkeit gehört. Es ist ja nicht zu übersehen, dass einige frühere neusachliche Maler sich nach 1933 dem Nationalsozialismus - und manchmal auch dessen nationalistischen Kunstidealen - angeschlossen bzw. angebiedert haben.

${ }^{51}$ Siehe dazu das Kapitel ,The cultural inheritance“ in Carus (2007, S. 41-64). 
Das hat etwa Olaf Peters (1998) eindrucksvoll gezeigt. ${ }^{52}$ Deswegen ist es naheliegend, hier noch einmal zu den Proponenten der Bewegung in der Architektur und Malerei, Roh und Giedion, zurückzugehen.

Dass Rohs Nach-Expressionismus sich durch einen betonten Internationalismus unterscheidet von der nur auf deutsche Malerei beschränkten Mannheimer Ausstellung „Neue Sachlichkeit“ von 1925, ist schon erwähnt worden. Er schreibt jedoch nur selten ausdrücklich von der neuen Kunstrichtung als einem internationalen Phänomen, etwa wenn es heißt:

Wir konstatieren zunächst nur, daß neben einer Reihe bestehender Arten von Malerei [wie dem Impressionismus und dem Expressionismus, Verf.) in allen Ländern Europas eine neue Art getreten sei. (Roh 1925, S. 3; meine Hervorhebung)

Für Roh ist es völlig selbstverständlich, in seinem Buch wie auch in anderen Veröffentlichungen die gesamte internationale Kunstszene der Gegenwart zu durchstreifen. Davon zeugt auch der Umstand, dass in seiner Liste von den 52 im Anhang des Buches genannten nachexpressionistischen Künstlern, die dort mit Abbildungen vertreten sind, weniger als die Hälfte Deutsche sind. ${ }^{53}$ Er ist noch in seinen spätesten Veröffentlichungen, wie etwa seinem Buch „Entartete“ Kunst: Kunstbarbarei im Dritten Reich aus dem Jahre 1962, auf die Unsinnigkeit nationalistischer Kunstauffassungen zurückgekommen, die in der Zwischenzeit durch den Nationalsozialismus besonders propagiert worden waren. So schrieb er etwa im Lichte dieser späteren Erfahrungen: ${ }^{54}$

Auch die Nationalsozialisten machten gewisse Grenzen des Gestaltens gerade zur bewußten Forderung, womit der Künstler eingeengt und zu geistiger Inzucht verführt wurde. Es ist nicht wahr, daß die Kultur und Kunst immer dann am höchsten stand, wenn sie ohne (auch noch so gut verarbeitete) Fremdeinflüsse blieb. Eine Internationalität der Kunst, welche die Hitlerleute so empörte, war gar kein Novum, sondern fand sich schon in Mittelalter und Barock. (Roh 1962, S. 120, Hervorhebungen im Original)

Giedion, wie Roh ja studierter Kunsthistoriker, hatte schon vor Hartlaubs epochemachender Ausstellung 1925 in Mannheim vorgehabt, in Zürich dasselbe Thema vorzustellen, nun allerdings mit internationaler Beteiligung. Dazu ist es aber - aus mir unbekannten Gründen - nicht gekommen. Danach konzentrierte er sich mehr und mehr auf die zeitgenössische Architektur.

\footnotetext{
${ }^{52}$ Allerdings scheint seine Stichprobe zu sehr auf die These einer Kontinuität der Neuen Sachlichkeit mit dem Nationalsozialismus zugeschnitten zu sein. Es ist jedoch keineswegs so, dass nennenswerte Anzahlen der früheren Neusachlichen zu den Nazis übergelaufen wären. Der Hofmaler der Nazis, Adolf Ziegler, den Peters als Beispiel für einen nationalsozialistischen Neusachlichen einschließt, wird in Rohs Buch Nach-Expressionismus in der Tat erwähnt (Roh 1925, S. 134). Es scheint aber, dass er in der Mitte der 1920er-Jahre noch einen anderen Stil bevorzugte als den monumentalen und heroischen der Nazi-Zeit.

${ }^{53}$ Roh (1925, S. 133 f.). Bei den 60 weiteren dort genannten Künstlern, die „,entweder schon seit Jahren in Richtung des Nachexpressionismus arbeiten oder sich in letzter Zeit diesen Zielen zuwandten“, sind die Deutschen in der Mehrzahl.

${ }^{54}$ Für seine Kritik an nationalistischen Tendenzen in der Literatur des Nationalsozialismus und bei ihren Protagonisten siehe Roh (1962, S. 85, 89).
} 
Bei Giedion heißt es schon in seinem zeitgeschichtlich orientierten ersten architekturtheoretischen Werk Bauen in Frankreich:

Wir wollen eine internationale Architektur. Eine Architektur der Zeit. Alle lebendigen Länder sind auf dem Wege zu ihr. Trotzdem hat jedes Land seine vorbestimmte Rolle in der Bewegung. Dies steht schon heute fest. (Giedion 2000 [1928], S. 68)

und, noch auf derselben Seite:

Wir dürfen es nicht unterlassen, nochmals hinzuzufügen: Die Kampffront national-international besteht in Wirklichkeit nicht mehr, so wie heute im Grunde die äußere Kampffront nicht zwischen den Staaten verläuft, sondern in einem durchgehenden Kampf um die soZIOLOGISCHE STRUKTUR.

Wie in Worten schon aus seinem Buch hervorgeht, sich dann aber auch im Laufe des 2. CIAM-Kongresses in Frankfurt am Main im Oktober 1929 (unter der Ägide von Walter Gropius und Giedion) in Taten zeigte, handelte es sich bei diesem „Kampf um die soziologische Struktur“ vor allem um die Forderung, das Neue Bauen auf die dringenden Erfordernisse des Bauens für das Existenzminimum auszurichten (Dahms 2004).

Wie sieht es mit dem Internationalismus bei Carnap aus? Bei ihm ist es angesichts einer Ausbildung als Mathematiker und Physiker von vornherein kein Wunder, dass er internationalistisch eingestellt war. Denn in diesen Disziplinen ist eine nationalistische Wissenschaftsauffassung (wie etwa die „Deutsche Mathematik“ oder die „Deutsche Physik“ im Nationalsozialismus) eher die Ausnahme. Für Carnaps internationalistische Ausrichtung spricht darüber hinaus - wie schon eingangs erwähnt - auch sein Engagement für die Völker verbindende Kunstsprache Esperanto, die nur von wenigen führenden Wissenschaftlern (wie z. B. Louis Couturat oder Wilhelm Ostwald vor dem Ersten Weltkrieg) geteilt wurde (Dahms 2016, S. 168 f.).

Später arbeitete Carnap an vorderster Front für die Internationalisierung des Logischen Empirismus, wie z. B. als Mitorganisator des Internationalen PhilosophieKongresses in Prag 1934 und der 1935 in Paris beginnenden „International Congresses of Unified Science“" ${ }^{55}$ Er gehörte auch zu den Herausgebern der ab 1938 erscheinenden „International Encyclopedia of Unified Science“ und verfasste dafür zwei der ersten Beiträge (Carnap 1938a/1969; 1938b/1969).

\subsection{Schlussbemerkungen}

Warum muss man all das wissen oder wenigstens beachten? Man muss nicht, aber man sollte, wenn man sich für die Zeitgeschichte von Philosophie und Wissenschaft und den kulturellen und politischen Kontext interessiert, in dem sie gedeihen oder behindert und sogar unterdrückt werden.

\footnotetext{
${ }^{55}$ Siehe dazu die Einleitung der Herausgeber sowie Dahms (2018).
} 
Hier geht es nun um die Einordnung in den Zeitgeist der 20er- und 30er-Jahre und die Fragen,

- warum bestimmte Leute und Gruppen aus unterschiedlichen kulturellen Feldern Affinitäten hatten,

- warum andere sich feindlich gegenüberstanden (z. B. linke und rechte Kritiker der Neuen Sachlichkeit).

Eine solche Betrachtung könnte ein Lehrstück dafür liefern, was der Zeitgeist dieser Epoche eigentlich war (und was ein solcher Zeitgeist vielleicht auch heute noch ist): Es geht nicht um einen unsichtbaren und unwägbaren intellektuellen Äther, der die Kultur und Gesellschaft einer Epoche mysteriös durchdringt und in dieser seiner Sphäre weht, wie er will. Es handelt sich vielmehr um ein kompliziertes Beziehungsgeflecht von einzelnen Akteuren mit einer gemeinsamen Haltung, die untereinander im Kontakt standen (ohne das an irgendeiner Stelle publik gemacht zu haben). Ihre Arbeit konnte natürlich nur in dem Grade Wirksamkeit entfalten, als das Publikum auf ihre Resultate reagierte. Solche Interaktionen sind durchaus nachvollziehbar und (kunst-, architektur- sowie philosophie-) historischer Forschung zugänglich. Es wird Zeit, solche Expeditionen ins archivalische Neuland zu wagen, um der von Giedion so genannten „Methodengleiche“ auf die Spur zu kommen. Daraus könnte man lernen, wieso Philosophen - im Vollgefühl ihrer Übereinstimmung mit Kollegen aus anderen Bereichen der Kultur - zeitweise dazu tendierten, auch problematische Theorien anzunehmen bzw. vorschnell andere abzulehnen, wenn sie von Geistesschaffenden vorgetragen wurden, die nicht kompatibel mit ihrem kulturellen Umfeld waren.

\section{Unveröffentlichte Quellen}

Nachlass Siegfried Giedion (im gta-Archiv Zürich)

- Briefwechsel mit Rudolf Carnap

- „Institute for Contemporary History“, Project o.D. (1941?), Archiv-Nr. 43-S- 8-6 Nachlass Rudolf Carnap (Hillman Library Pittsburgh/Universität Konstanz)

- Tagebücher

- Vorträge am Bauhaus Dessau, Oktober 1929

Der Mißbrauch der Sprache (RC 110-07-43:1)

Aufgabe und Gehalt der Wissenschaft (RC 110-07-47)

- Vortrag am New Bauhaus, Chicago

Knowledge \& Action. The Task of Science, 16.05.1938 (RC 110-08-21:1)

- Briefwechsel mit

Wilhelm Flitner

Charles Morris

Ernest Nagel

- Sammlung Sera-Kreis (ohne Signatur), darin: 
Martha Hörmann: Sera, Sommer 1913

Friedenssonnenwende auf den Hohen Leeden 1919, Jena 1919

Franz Roh Papers (Getty Research Institute, Los Angeles)

- 850120 Correspondence, Rudolf Carnap (Box I, Folder 4)

Archiv der Kunsthalle Mannheim

- Briefwechsel Gustav Hartlaub mit

Franz Roh

Karl Rössing

\section{Literatur}

Albert, K. 1968. Philosophie der modernen Kunst. Meisenheim am Glan: Anton Hain.

Awodey, S., und C. Klein, Hrsg. 2004. Carnap brought home: The view from Jena. Chicago/LaSalle: Open court.

Beeck, K.-H. 1975. Friedrich Wilhelm Dörpfeld: Anpassung im Zwiespalt: Seine politisch-sozialen Auffassungen. Neuwied/Berlin: Luchterhand.

Bernhard, P. 2015. Neopositivismus und Neues Bauen: Zur Entdeckung einer ,inneren Verwandtschaft". In Architektur und Philosophie: Grundlagen. Standpunkte. Perspektiven, Hrsg. J.H. Gleiter und L. Schwarte. Bielefeld: transcript.

2021. Carnap und das Bauhaus, in diesem Band.

Bourdeau, M., G. Heinzmann, und P. Wagner Hrsg. 2018. Sur la philosophie scientifique et l'unité de la science: Le congrès de Paris 1935 et son héritage: Actes du colloque de Cerisy, Philosophia Scientiæ 22(3). Paris: Éditions Kimé

Buderer, H.-J. und M. Fath. 1994. Neue Sachlichkeit: Bilder auf der Suche nach der Wirklichkeit: Figurative Malerei der zwanziger Jahre, Hrsg. u. m. einem Vorw. v. M. Fath, Ausstellungskatalog Städtische Kunsthalle Mannheim 1994/1995. München/New York: Prestel.

Bühler, K. 1933. Die Axiomatik der Sprachwissenschaften. Kant-Studien 38(1/2): 19-90.

Carnap, R. 1922. Der Raum: Ein Beitrag zur Wissenschaftslehre, Kant-Studien, Ergänzungshefte 56. Berlin: Reuther u. Reichard.

1928. Der logische Aufbau der Welt. Hamburg: Felix Meiner.

1932. Überwindung der Metaphysik durch logische Analyse der Sprache. Erkenntnis 2(4): 219-241.

- 1938a. Logical foundations of the unity of science. Neurath/Carnap/Morris 1969:42-62

1938b. Foundations of logic and mathematics. Neurath/Carnap/Morris 1969:139-213

1963. Intellectual autobiography. In The philosophy of Rudolf Carnap, Hrsg. P.A. Schilpp, 3-84. LaSalle: Open Court.

Carus, A.W. 2007. Carnap and twentieth-century thought: Explication as enlightenment. Cambridge: Cambridge University Press.

Czech, H., und W. Mistelbauer. 1984. Das Looshaus. Wien: Löcker.

Dahms, H.-J. 1996. Vienna circle and French enlightenment - A comparison of diderots Encyclopédie with Neuraths international encyclopedia of unified science. In Encyclopedia and 3l. The life and work of Otto Neurath (1882-1845), Hrsg. Elisabeth Nemeth und Friedrich Stadler, 53-61. Dordrecht/Boston/London: Kluwer Academic Publishers.

2001. Neue Sachlichkeit in der Architektur und Philosophie der 20er Jahre. Arch+: Z Architektur und Städtebau 34:82-87. 
2002. Mies van der Rohe und die Philosophie um 1930. Arch+: ZArchitektur und Städtebau 161. http://www.archplus.net/archiv_artikel.php. Zugegriffen am 30.12.2020.

—. 2003. 1929 - CIAM-Kongress: Die Wohnung für das Existenzminimum: Verwissenschaftlichung und Formverzicht. In Bauhausstil: Zwischen International Style und Lifestyle, Hrsg. R. Bittner, 86-107. Berlin: Jovis.

- 2004. Neue Sachlichkeit in the architecture and philosophy of the 1920s. Awodey/Klein 2004:357-375.

- 2016. Carnap's early conception of a "system of the sciences": The importance of Wilhelm Ostwald. In Influences on the Aufbau, Hrsg. C. Damböck, 163-185. Cham/Heidelberg/ New York/Dordrecht/London: Springer.

- 2018. Mission accomplished? Unified science and logical empiricism at the 1935 Paris Congress and afterwards. Bourdeau/Heinzmann/Wagner 2018:289-305.

Findeli, A. 1995. Le Bauhaus de Chicago: L’œuvre pédagogique de László Moholy-Nagy. Québec: Sillery.

Flitner, W. 1986. Erinnerungen 1889-1945, Gesammelte Schriften 11. Paderborn/München/Wien/ Zürich: F. Schöningh.

Galison, P. 1990. Aufbau/Bauhaus: Logical positivism and architectural modernism. Critical Inquiry 16:709-752.

Georgiadis, S. 1989. Sigfried Giedion: Eine intellektuelle Biographie. Zürich: Ammann.

Giedion, S. 1922. Spätbarocker und romantischer Klassizismus. München: F. Brückmann. - 1928/2000. Bauen in Frankreich, Bauen in Eisen, Bauen in Eisenbeton. Leipzig/Berlin:

Klinkhardt \& Biermann, 1928. Neuaufl. Berlin: Gebr. Mann, 2000 (m. einem Nachw. v. S. Georgiadis).

_- 1929. Neue Optik (Abstrakte und surrealistische Malerei und Plastik; Ausstellung im Kunsthaus Zürich 1929). Giedion 1987:43-50.

—. 1931. Erziehung zum Sehen (Vortrag f. d. Davoser Hochschulkurse). Giedion 1987:18-22. 1941. Space, time and architecture: The growth of a new tradition. Cambridge, MA: Harvard University Press.

- 1987. Wege in die Öffentlichkeit: Aufsätze und unveröffentlichte Schriften aus den Jahren 1926-1956, Hrsg. u. komm. v. D. Huber. Zürich: GTA, Institut für Geschichte und Theorie der Architektur ETH.

Goebel, K. 1970. Die Wuppertaler Familie Carnap. Romerike Berge 20(1): 9-14.

Graumann, C.F., und T. Herrmann, Hrsg. 1984. Karl Bühlers Axiomatik: 50 Jahre Axiomatik der Sprachwissenschaften. Frankfurt a. M.: Klostermann.

Haller, R., und F. Stadler, Hrsg. 1993. Wien-Berlin-Prag: Der Aufstieg der wissenschaftlichen Philosophie: Zentenarien Rudolf Carnap - Hans Reichenbach - Edgar Zilsel, Veröffentlichungen des Instituts Wiener Kreis 2. Wien: Hölder-Pichler-Tempsky.

Heidegger, M. 1929. Was ist Metaphysik? Frankfurt a. M.: Vittorio Klostermann.

Horkheimer, M. 1937. Der neueste Angriff auf die Metaphysik. Zeitschrift für Sozialforschung 6:4-51.

Kirsch, K. 1987. Die Weißenhofsiedlung. Werkbund Ausstellung „Die Wohnung “. Stuttgart: Deutsche Verlags-Anstalt.

Krischanitz, A., and O. Kapfinger. 1985. Die Wiener Werkbundsiedlung: Dokumentation einer Erneuerung. Wien: Beton.

Kruft, H.-W. 1995. Geschichte der Architekturtheorie: Von der Antike bis zur Gegenwart. 4. Aufl. München: C. H. Beck.

Limbeck-Lilienau, C. 2010. Rudolf Carnap und die Philosophie in Amerika. Logischer Empirismus, Pragmatismus, Realismus. In Vertreibung, Transformation und Rückkehr der Wissenschaftstheorie. Am Beispiel von Rudolf Carnap und Wolfgang Stegmüller, Hrsg. F. Stadler, 85-164. Wien: LIT

. 2015. Der Wiener Kreis: Eine illustrierte Geschichte des Logischen Empirismus. LimbeckLilienau/Stadler 2015:357-411. 
Limbeck-Lilienau, C., und F. Stadler. 2015. Der Wiener Kreis: Texte und Bilder zum Logischen Empirismus. Wien: LIT.

Loos, A. 1908/1982. Ornament und Verbrechen. In Trotzdem: Gesammelte Schriften 1900-1930, Hrsg. ders, 78-88. Wien: Prachner.

Loos, A. 1983. Die Potemkische Stadt. Verschollene Schriften 1897-1933 (Hrsg. Adolf Opel). Wien: Georg Prachner.

Meyer, H. 1980. Bauen und Gesellschaft: Schriften, Briefe, Projekte, Hrsg. v. L. Meyer-Bergner, bearb. u. m. Einführungen versehen v. K.-J. Winkler. Dresden: Verlag der Kunst.

_. 1926/1989. Die neue Welt. Abg. In Hannes Meyer 1889-1954: Architekt, Urbanist, Lehrer, Hrsg. Bauhaus-Archiv Berlin, Deutsches Architekturmuseum Frankfurt am Main, 70-73. Berlin: Ernst \& Sohn.

Moholy-Nagy, L. 1927/1967. Malerei, Fotografie, Film. München: Albert Langen. Nachdr. Kupferberg, Mainz/Berlin 1967 (= Neue Bauhausbücher). 1929/1968. Von Material zu Architektur. ..., München. Nachdr. Mainz/Berlin (= Neue Bauhausbücher)

Mück, H.-D., et al. 2000. Magie der Realität, Magie der Form: Eine Hommage für Franz Roh 1890-1965, Katalogbuch zur Ausstellung in Apolda 16.01.-12.03.2000. Apolda: ARTeFACT.

Neurath, O. 1921. Anti-Spengler. München: Georg D. W. Callwey. . 1931a. Physikalismus: Die Philosophie des Wiener Kreises. Neurath 1981:413-421. 1931b. Empirische Soziologie: Der wissenschaftliche Gehalt der Geschichte und der Nationalökonomie. Neurath 1981:423-517.

- 1981. Gesammelte philosophische und methodologische Schriften (2 Bde), Hrsg. v. R. Haller u. H. Rutte. Wien: Hölder-Pichler-Tempsky.

Neurath, O., R. Carnap, und C. Morris. 1969. Foundations of the unity of science: Toward an international encyclopedia of unified science (2 Bde). Chicago: University of Chicago Press.

Niemeyer, O. 1998. As curvas do tempo: Memórias. Rio de Janeiro: Editora Revan.

- 2005. Minha Arquitetura 1937-2005. Rio de Janeiro: Editora Revan.

Peters, O. 1998. Neue Sachlichkeit und Nationalsozialismus: Affirmation und Kritik 1931-1947. Berlin: Dietrich Reimer.

Ratzke, E. 1987. Das Pädagogische Institut der Universität Göttingen: Ein Überblick über seine Entwicklung in den Jahren 1923-1949. In Die Universität Göttingen unter dem Nationalsozialismus: Das verdrängte Kapitel ihrer 250jährigen Geschichte, Hrsg. H. Becker, H.-J. Dahms, und C. Wegeler, 200-218. München etc.: K. G. Saur.

Roh, F. 1921. Holländische Malerei. Jena: Diederichs. . 1925. Nach-Expressionismus: Magischer Realismus: Probleme der neuesten europäischen Malerei. Leipzig: Klinkhardt \& Biermann. . 1962. „Entartete“ Kunst: Kunstbarbarei im Dritten Reich. Hannover: Fackelträger.

Schmied, W. 1969. Neue Sachlichkeit und Magischer Realismus in Deutschland 1918-1933. Hannover: Fackelträger.

Stadler, F. Hrsg. 1993. Scientific philosophy: Origins and developments. Dordrecht: Springer. 1995. Wissenschaftliche Weltauffassung und Kunst: Zur werttheoretischen Dimension im Wiener Kreis. Deutsche Zeitschrift für Philosophie 43:635-651.

Thiel, C. 1993. Carnap und die wissenschaftliche Philosophie auf der Erlanger Tagung 1923. Haller/Stadler 1993:175-188.

Verein Ernst Mach. 1929/1981. Wissenschaftliche Weltauffassung: Der Wiener Kreis. A. Wolf, Wien. Abg. Neurath 1981:299-336.

Welzig, M. 1998. Josef Frank (1885-1967): Das architektonische Werk. Wien/Köln/Weimar: Böhlau.

Werner, M.G. 2003. Moderne in der Provinz: Kulturelle Experimente im Fin de Siècle Jena. Göttingen: Wallstein. 
Winkler, K.-J. 1989. Der Architekt Hannes Meyer: Anschauungen und Werk. Berlin: Verlag für Bauwesen.

Zilsel, E. 1918/1990. Die Geniereligion: Ein kritischer Versuch über das moderne Persönlichkeitsideal, mit einer historischen Begründung. Wien/Leipzig/Frankfurt a. M.: Braumüller/ Suhrkamp.

Open Access This chapter is licensed under the terms of the Creative Commons Attribution 4.0 International License (http://creativecommons.org/licenses/by/4.0/), which permits use, sharing, adaptation, distribution and reproduction in any medium or format, as long as you give appropriate credit to the original author(s) and the source, provide a link to the Creative Commons license and indicate if changes were made.

The images or other third party material in this chapter are included in the chapter's Creative Commons license, unless indicated otherwise in a credit line to the material. If material is not included in the chapter's Creative Commons license and your intended use is not permitted by statutory regulation or exceeds the permitted use, you will need to obtain permission directly from the copyright holder. 